hep-th/0410217

ITP-UH-24/04

UUITP-24/04

HIP-2004-54/TH

\title{
T-duality for the sigma model with boundaries
}

\author{
Cecilia Albertsson $^{a 1}$, Ulf Lindström ${ }^{b 2}$ and Maxim Zabzine ${ }^{c 3}$ \\ ${ }^{a}$ Institut für Theoretische Physik, Universität Hannover, \\ Appelstraße 2, DE-30167 Hannover, Germany \\ ${ }^{b}$ Department of Theoretical Physics, Uppsala University, \\ Box 803, SE-751 08 Uppsala, Sweden \\ and \\ HIP - Helsinki Institute of Physics \\ P.O. Box 64 FIN-00014 University of Helsinki, Suomi - Finland \\ ${ }^{c}$ School of Mathematical Sciences, Queen Mary, University of London, \\ Mile End Road, London, E1 4NS, UK
}

\begin{abstract}
We derive the most general local boundary conditions necessary for T-duality to be compatible with superconformal invariance of the two-dimensional $\mathcal{N}=1$ supersymmetric nonlinear sigma model with boundaries. To this end, we construct a consistent gauge invariant parent action by gauging a $U(1)$ isometry, with and without boundary interactions. We investigate the behaviour of the boundary conditions under T-duality, and interpret the results in terms of D-branes.
\end{abstract}

\footnotetext{
${ }^{1}$ e-mail address: cecilia@itp.uni-hannover.de

${ }^{2}$ e-mail address: ulf.lindstrom@teorfys.uu.se

${ }^{3}$ e-mail address: m.zabzine@qmul.ac.uk
} 


\section{Introduction}

Classical T-duality can be realised as a transformation acting on the worldsheet fields in the two-dimensional nonlinear sigma model. This realisation is straightforward when there is an isometry of the target space which at the same time is a symmetry of the worldsheet action. By gauging the isometry (making it locally dependent on the worldsheet coordinates) one obtains a gauge invariant "parent action" from which the original and dual actions are extracted by integrating out different fields $[1,2,3]$. In the process one finds the transformation on the worldsheet fields as well as Buscher's rules $[4,5]$ which yield the dual background.

The simplest situation occurs when the isometry group is abelian. In this case the duality transformation is symmetric in the sense that one can start from either theory (the original or the dual one) and obtain the other one via the same gauging process [6]. But for nonabelian isometries the dual model does not possess the same nonabelian isometry as the original theory, rendering the gauging procedure less straightforward [7]. In such a situation, one obtains the dual model by gauging a subgroup of the isometry, but a corresponding gauging of the dual model does not bring you back to the original model.

T-duality by way of gauging isometries has been extensively studied for the nonlinear sigma model $[8,9,10]$. In particular closed string T-duality in the presence of a $U(1)$ isometry is well understood, both for the bosonic model and the models with $\mathcal{N}=1$ and $\mathcal{N}=2$ worldsheet supersymmetry $[11,12,13,14]$. The operative point in those investigations is that the parent action is gauge invariant up to boundary terms, if the background is subject to certain constraints. For the open string, however, the worldsheet has boundaries, and the gauging procedure must be performed with care so as to make the parent action gauge invariant also on the boundary. In the bosonic case this is achieved by imposing the appropriate boundary conditions on the worldsheet fields and on the added gauge fields. But the supersymmetric action in general receives an extra boundary term $[15,16]$, necessitating an addition of gauge fields on the boundary. These additional boundary terms affect the resulting boundary conditions.

The structure of the paper is as follows. In Section 2 we review the $\mathcal{N}=1$ supersymmetric nonlinear sigma model with boundaries and the set of boundary conditions required for superconformal invariance. Assuming there is a group acting on the target space we derive in Section 3 the conditions necessary for the model to be invariant under the action of that group, first for the bosonic case and then for the supersymmetric one. We find that the symmetry, when it exists, is global (i.e., the infinitesimal parameter of the isometry transformation is independent of the worldsheet coordinates), and that the group is an isometry of the target space metric. The derivation involves two invariance checks: the action and the 
boundary conditions. Armed with the full set of boundary conditions for superconformal and isometry invariance of the original model, we then construct the appropriate parent action, first the bosonic one in Section 4, and then the $\mathcal{N}=1$ supersymmetric one in Section 5. For each parent action we derive the conditions necessary for a consistent retrieval of the original and dual models. Gauge invariance is checked for the action, the field equations, and for the boundary conditions themselves. We moreover verify that the supersymmetric parent action is supersymmetric also on the boundary, given the most general classical ansatz for the fermionic boundary conditions. In Section 6 we add boundary interactions to the sigma model, showing how to incorporate them in the T-duality transformation. The model dual to a superconformal model is expected to also be superconformal, hence the worldsheet fields of the dual model must obey the corresponding superconformality conditions. We briefly discuss in Section 7 how these $\mathcal{N}=1$ conditions transform under T-duality. Finally, Section 8 contains our conclusions and a wishlist for future research.

\section{Superconformal boundary conditions}

Here we give a summary of the derivation in [16] of boundary conditions for the $\mathcal{N}=1$ supersymmetric nonlinear sigma model. ${ }^{4}$ This model is given by the following action,

$$
S=\int d^{2} \xi d^{2} \theta D_{+} \Phi^{\mu} D_{-} \Phi^{\nu} E_{\mu \nu}(\Phi)+\frac{i}{2} \int d \tau B_{\mu \nu}\left[\psi_{+}^{\mu} \psi_{+}^{\nu}+\psi_{-}^{\mu} \psi_{-}^{\nu}\right]
$$

where $E_{\mu \nu} \equiv g_{\mu \nu}+B_{\mu \nu}$ denotes a background of general (Riemannian) metric $g_{\mu \nu}$ and an antisymmetric two-tensor $B_{\mu \nu}$ with field strength $H_{\mu \nu \rho}=\frac{1}{2} \partial_{[\mu} B_{\nu \rho]}$. The boundary term is required for $\mathcal{N}=1$ supersymmetry to be preserved on the boundary. In component form (2.1) reads (for notation, see Appendix A)

$$
\begin{aligned}
S= & \int_{\Sigma} d^{2} \xi\left[\partial_{+} X^{\mu} \partial_{-} X^{\nu} E_{\mu \nu}+i \psi_{+}^{\mu} \nabla_{-}^{(+)} \psi_{+}^{\nu} g_{\mu \nu}+i \psi_{-}^{\mu} \nabla_{+}^{(-)} \psi_{-}^{\nu} g_{\mu \nu}\right. \\
& \left.+\frac{1}{2} \psi_{+}^{\lambda} \psi_{+}^{\sigma} \psi_{-}^{\rho} \psi_{-}^{\gamma} \mathcal{R}_{\rho \gamma \lambda \sigma}^{-}\right]
\end{aligned}
$$

where the worldsheet coordinates are $\tau \in \mathbb{R}$ and $\sigma \in[0, \pi]$, and the curvature $\mathcal{R}_{\rho \gamma \lambda \sigma}^{ \pm}$is defined as

$$
\mathcal{R}_{\sigma \rho \lambda}^{ \pm \mu}=\Gamma_{\lambda \sigma, \rho}^{ \pm \mu}-\Gamma_{\rho \sigma, \lambda}^{ \pm \mu}+\Gamma_{\rho \gamma}^{ \pm \mu} \Gamma_{\lambda \sigma}^{ \pm \gamma}-\Gamma_{\lambda \gamma}^{ \pm \mu} \Gamma_{\rho \sigma}^{ \pm \gamma}
$$

with $\Gamma_{\nu \rho}^{ \pm \mu}$ given by

$$
\Gamma_{\rho \sigma}^{ \pm \nu} \equiv \Gamma_{\rho \sigma}^{\nu} \pm g^{\nu \mu} H_{\mu \rho \sigma},
$$

\footnotetext{
${ }^{4}$ The $\mathcal{N}=2$ supersymmetric nonlinear sigma model was treated in $[17,18,19]$.
} 
and $\nabla_{ \pm}^{( \pm)}$by

$$
\nabla_{ \pm}^{(+)} \psi_{+}^{\nu}=\partial_{\underline{\underline{\#}}} \psi_{+}^{\nu}+\Gamma_{\rho \sigma}^{+\nu} \partial_{\underline{\underline{\#}}} X^{\rho} \psi_{+}^{\sigma}, \quad \nabla_{ \pm}^{(-)} \psi_{-}^{\nu}=\partial_{\underline{\underline{\#}}} \psi_{-}^{\nu}+\Gamma_{\rho \sigma}^{-\nu} \partial_{\underline{\underline{\#}}} X^{\rho} \psi_{-}^{\sigma}
$$

In $(2.2)$ we have used the equations of motion for the auxiliary field $F_{+-}^{\mu}$,

$$
F_{+-}^{\rho}+\Gamma_{\lambda \sigma}^{-\rho} \psi_{+}^{\lambda} \psi_{-}^{\sigma}=0
$$

To find the supersymmetric boundary conditions from (2.1), we require the boundary terms in the Euler-Lagrange equations to vanish simultaneously with those in the supersymmetry variation. The two boundary variations read (again using (2.6))

$$
\begin{aligned}
\delta S= & i \int d \tau\left[\left(\delta \psi_{+}^{\mu} \psi_{+}^{\nu}-\delta \psi_{-}^{\mu} \psi_{-}^{\nu}\right) g_{\mu \nu}\right. \\
& \left.+\delta X^{\mu}\left(i \partial_{+} X^{\nu} E_{\nu \mu}-i \partial_{=} X^{\nu} E_{\mu \nu}+\Gamma_{\nu \mu \rho}^{-} \psi_{-}^{\nu} \psi_{-}^{\rho}-\Gamma_{\nu \mu \rho}^{+} \psi_{+}^{\nu} \psi_{+}^{\rho}\right)\right], \\
\delta_{s} S= & \epsilon^{-} \int d \tau\left[\partial_{+} X^{\mu} \psi_{-}^{\nu} E_{\mu \nu}-\eta \psi_{+}^{\mu} \partial_{=} X^{\nu} E_{\mu \nu}+\eta \partial_{+} X^{\mu} \psi_{+}^{\nu} B_{\mu \nu}+\partial_{=} X^{\mu} \psi_{-}^{\nu} B_{\mu \nu}\right. \\
& \left.-\frac{i}{3} \eta H_{\mu \nu \rho} \psi_{+}^{\rho} \psi_{+}^{\mu} \psi_{+}^{\nu}-\frac{i}{3} H_{\mu \nu \rho} \psi_{-}^{\rho} \psi_{-}^{\mu} \psi_{-}^{\nu}\right] .
\end{aligned}
$$

Since the boundary relates left- and right-movers, we make a general ansatz for the relation between $\psi_{+}^{\mu}$ and $\psi_{-}^{\mu}$. The goal is then to find the restrictions on this ansatz arising from $\delta S=\delta_{s} S=0$. The most general local fermionic boundary condition ${ }^{5}$ allowed (classically) by dimensional analysis is of the simple form

$$
\psi_{-}^{\mu}=\eta R_{\nu}^{\mu}(X) \psi_{+}^{\nu},
$$

where $R^{\mu}{ }_{\nu}(X)$ is a locally defined object which transforms as a $(1,1)$ tensor field under coordinate transformations, and $\eta= \pm 1$ corresponds to the choice of spin structures. The bosonic counterpart of (2.9) is obtained by means of a supersymmetry transformation, ${ }^{6}$ and reads

$$
\partial_{=} X^{\mu}-R_{\nu}^{\mu} \partial_{+} X^{\nu}+2 i\left(P_{\rho}^{\sigma} \nabla_{\sigma} R_{\nu}^{\mu}+P_{\gamma}^{\mu} g^{\gamma \delta} H_{\delta \sigma \rho} R_{\nu}^{\sigma}\right) \psi_{+}^{\rho} \psi_{+}^{\nu}=0,
$$

where $P_{\nu}^{\mu} \equiv\left(\delta_{\nu}^{\mu}+R_{\nu}^{\mu}\right) / 2$. The conditions (2.9) and (2.10) satisfy $\delta S=\delta_{s} S=0$ if and only if $R^{\mu}{ }_{\nu}$ obeys

$$
\begin{gathered}
g_{\rho \sigma}=R_{\rho}^{\mu} g_{\mu \nu} R_{\sigma}^{\nu} \\
P_{\tau}^{\rho} R_{\sigma}^{\mu} g_{\mu \nu} \nabla_{\rho} R_{\gamma}^{\nu}+P_{\sigma}^{\rho} R_{\gamma}^{\mu} g_{\mu \nu} \nabla_{\rho} R_{\tau}^{\nu}+P_{\gamma}^{\rho} R_{\tau}^{\mu} g_{\mu \nu} \nabla_{\rho} R_{\sigma}^{\nu}+4 P_{\tau}^{\mu} P_{\sigma}^{\nu} P_{\gamma}^{\rho} H_{\mu \nu \rho}=0 .
\end{gathered}
$$

\footnotetext{
${ }^{5}$ In this paper we only consider local boundary conditions for the $(X, \psi)$ fields. Non-local boundary conditions for bosonic sigma models and their properties under T-duality have been considered in [20].

${ }^{6}$ For an explicit component form of this transformation, see Eq. (A.4).
} 


\subsection{Interpretation}

These constraints imply some interesting geometrical properties of the boundary. To make these properties precise, we define an operator that projects vectors onto the Dirichlet directions, and we call it the Dirichlet projector $Q^{\mu}{ }_{\nu}$ (see, e.g., [21]). It satisfies $Q^{2}=Q$ and

$$
R_{\rho}^{\mu} Q_{\nu}^{\rho}=Q_{\rho}^{\mu} R_{\nu}^{\rho}=-Q_{\nu}^{\mu}
$$

The relations (2.13) are motivated by the expectation that the Dirichlet condition on the fermions reads (see, e.g., [22])

$$
\psi_{-}^{i}+\eta \psi_{+}^{i}=0
$$

where $i$ labels the Dirichlet directions in some chosen coordinate system. In covariant form it reads

$$
Q_{\nu}^{\mu}\left(\psi_{-}^{\nu}+\eta \psi_{+}^{\nu}\right)=0
$$

The projector complementary to $Q^{\mu}{ }_{\nu}$ is the Neumann projector $\pi_{\nu}^{\mu} \equiv \delta_{\nu}^{\mu}-Q^{\mu}{ }_{\nu}$, which projects vectors onto the Neumann directions and which satisfies

$$
\pi_{\rho}^{\mu} P_{\nu}^{\rho}=P_{\rho}^{\mu} \pi_{\nu}^{\rho}=P_{\nu}^{\mu} .
$$

Then, by contracting (2.11) and (2.12) with $Q_{\lambda}^{\tau}$, we find the conditions

$$
\pi_{\rho}^{\mu} g_{\mu \nu} Q_{\sigma}^{\nu}=0
$$

and

$$
\pi_{\gamma}^{\mu} \pi_{\nu}^{\rho} \pi_{[\mu, \rho]}^{\delta}=0
$$

In a suitably chosen coordinate system (2.17) implies the diagonalisation of the metric, in a sense decoupling Neumann from Dirichlet directions. Condition (2.18) is the integrability condition for $\pi_{\nu}^{\mu}$, implying that the D-brane must be a maximal integral submanifold of the target space $[23,24]$.

Next we contract (2.10) with $Q^{\mu}{ }_{\nu}$, and use (2.18), to obtain

$$
Q_{\nu}^{\mu} \partial_{\tau} X^{\nu}=0
$$

This is just the Dirichlet condition for the end of the string, confining it to the brane defined by $\pi_{\nu}^{\mu}$. Furthermore, by contracting Eq. (2.12) with $\pi_{\nu}^{\mu}$ one finds

$$
\pi_{\mu}^{\rho} E_{\sigma \rho} \pi_{\nu}^{\sigma}-\pi_{\mu}^{\rho} E_{\rho \sigma} \pi_{\lambda}^{\sigma} R_{\nu}^{\lambda}=0
$$

If (2.20) holds, then condition (2.12) is just the statement that the field strength of the B-field on the brane coincides with the pullback of the background field strength $H_{\mu \nu \rho}$ to the brane. Note that for a spacefilling brane (when all directions are Neumann), Eq. (2.20) implies, schematically, $R=E^{-1} E^{t}$. 


\subsection{D superfield formalism}

The boundary ansatz (2.9) and its superpartner (2.10) are easier to handle in 1D superfield formalism than in component form. In the notation of Appendix B, the two conditions can be written as a single equation, as follows (see also [25]),

$$
\left(D K^{\mu}+S^{\mu}\right)=R_{\nu}^{\mu}(K)\left(D K^{\nu}-S^{\nu}\right),
$$

where the $1 \mathrm{D}$ superderivative $D$ satisfies $D^{2}=i \partial_{\tau}$. Contracting $(2.21)$ with $Q^{\mu}{ }_{\nu}$ we find

$$
Q_{\nu}^{\mu}(K) D K^{\nu}=0
$$

which encodes the two Dirichlet conditions (2.15) and (2.19). It is equivalent to

$$
D K^{\mu}=\pi_{\nu}^{\mu}(K) D K^{\nu} .
$$

We can try to deduce some information also about $S^{\mu}$, by solving for it in (2.21). The result is

$$
2 P_{\rho}^{\mu} \pi_{\nu}^{\rho} S^{\nu}=-\left(\delta_{\nu}^{\mu}-R_{\nu}^{\mu}\right) D K^{\nu}
$$

implying that, in the presence of a B-field, $\pi^{\mu}{ }_{\nu} S^{\nu} \neq 0$, so $S^{\mu}$ has a nonzero Neumann part. When the B-field vanishes, however, we see that $\pi^{\mu}{ }_{\nu} S^{\nu}=0$, because then it follows from (2.20) that $\left(\delta_{\nu}^{\mu}-R_{\nu}^{\mu}\right) \pi_{\rho}^{\nu}=0$.

\section{$2.3 \quad$ Summary}

$$
\begin{aligned}
g_{\rho \sigma}-R^{\mu}{ }_{\rho} g_{\mu \nu} R^{\nu}{ }_{\sigma} & =0 \\
R^{\mu}{ }_{\rho} Q^{\rho}{ }_{\nu}=Q^{\mu}{ }_{\rho} R^{\rho}{ }_{\nu} & =-Q^{\mu}{ }_{\nu} \\
\pi^{\mu}{ }_{\rho} g_{\mu \nu} Q^{\nu}{ }_{\sigma} & =0 \\
\pi^{\mu}{ }_{\gamma} \pi^{\rho}{ }_{\nu} \pi^{\delta}{ }_{[\mu, \rho]} & =0 \\
\pi_{\mu}^{\rho} E_{\sigma \rho} \pi_{\nu}^{\sigma}-\pi^{\rho}{ }_{\mu} E_{\rho \sigma} \pi^{\sigma}{ }_{\lambda} R^{\nu}{ }_{\nu} & =0 \\
Q^{\mu}{ }_{\nu}(K) D K^{\nu} & =0
\end{aligned}
$$

Table 1: Boundary conditions for $\mathcal{N}=1$ superconformal invariance of the nonlinear sigma model, listed in order of appearance. We have assumed the ansatz (2.21).

Given the ansatz (2.21) we summarise in Table 1 the boundary conditions necessary for $\mathcal{N}=1$ superconformal invariance of the nonlinear sigma model. These conditions may alternatively be obtained by considering the currents corresponding to superconformal invariance on the boundary $[16,26]$. 
Remark: We collect conditions in tables such as this, at the end of the sections where we derive them. In most cases any boundary condition referred to in the text can be found in one of those tables.

\section{$3 \quad$ Isometry}

Let $M$ be a manifold with metric $g$ and local coordinates $X^{\mu}$. Let the group $G$ act on $M$, and let the group action be realised at the infinitesimal level by vector fields $k_{A}^{\mu}(X)$ which generate the corresponding Lie algebra,

$$
\left[k_{A}, k_{B}\right]=f_{A B}^{C} k_{C}
$$

where $f_{A B}{ }^{C}$ are the structure constants. Under the action of $G$ the coordinates transform as

$$
\delta_{k} X^{\mu}=\epsilon^{A} k_{A}^{\mu}(X)
$$

where $\epsilon^{A}$ is the infinitesimal parameter of the transformation, independent of $X^{\mu}$. When $M$ is the target space of the nonlinear sigma model, we want to know the conditions necessary for $G$ to be a symmetry of the model.

First note that $k_{A}^{\mu}$ is a Neumann vector,

$$
Q_{\nu}^{\mu} k_{A}^{\nu}=0
$$

a fact we use frequently in this paper. This can be seen from the Dirichlet condition in a suitably chosen coordinate system where $X^{n}$ label Neumann directions and $X^{i}$ are Dirichlet directions,

$$
\partial_{\tau} X^{i}=0
$$

the solution of which describes a foliation,

$$
X^{i}=c^{i}
$$

for some constants $c^{i}$. In the supersymmetric model, where the Neumann projector $\pi_{\nu}^{\mu}$ is integrable, each constant describes an integral submanifold. There are now two different questions to ask regarding invariance under the group $G$. First, when is the foliation as a whole invariant, i.e., when is Eq. (3.3) invariant? Second, when is each individual submanifold invariant, i.e., when is Eq. (3.4) invariant for any chosen constant $c^{i}$ ? The first question will be answered in what follows. To answer the second question, we apply the variation $\delta_{k} X^{i}=\epsilon^{A} k_{A}^{i}$ to (3.4) and find

$$
k_{A}^{i}=0
$$


the covariant version of which is Eq. (3.2).

We first analyse the bosonic model and then the supersymmetric one, in each case checking invariance of the action and of any relevant boundary conditions. Invariance of the field equations should follow from that of the action. Nevertheless, as a check, we have verified that this is indeed the case.

\subsection{Bosonic model}

\subsubsection{Action}

The variation of the bosonic action

$$
S_{\mathrm{bos}}=\int d^{2} \xi \partial_{+} X^{\mu} \partial_{=} X^{\nu} E_{\mu \nu}
$$

under (3.1) is given by

$$
\begin{aligned}
\delta_{k} S_{\text {bos }}= & \int d^{2} \xi\left[\epsilon^{A} \partial_{+} X^{\mu} \partial_{=} X^{\nu} \mathcal{L}_{k_{A}} E_{\mu \nu}+\partial_{+} \epsilon^{A} \partial_{=} X^{\mu} k_{A}^{\nu} E_{\nu \mu}\right. \\
& \left.+\partial_{+} X^{\mu} \partial_{=} \epsilon^{A} k_{A}^{\nu} E_{\mu \nu}\right] .
\end{aligned}
$$

If the parameter $\epsilon^{A}$ is constant, i.e., $\partial_{\#} \epsilon^{A}=0$, then (3.6) vanishes up to a boundary term if the Lie derivative (defined in Appendix $\mathrm{C}$ ) of the metric with respect to $k_{A}^{\mu}$ satisfies

$$
\mathcal{L}_{k_{A}} g_{\mu \nu}=0
$$

and that of the B-field satisfies

$$
\mathcal{L}_{k_{A}} B_{\mu \nu}=\omega_{A[\nu, \mu]}
$$

for some one-form $\omega_{A}$. Eq. (3.7) is equivalent to invariance of the metric under (3.1), meaning that the transformation is an isometry and $k_{A}^{\mu}$ are Killing vectors. In the special case with a single Killing vector lying along the $X^{0}$-direction $\left(k_{A}^{\mu}=\delta_{0}^{\mu}\right)$, this condition reads $g_{\mu \nu, 0}=0$, i.e., the metric is independent of the isometry direction. In compliance with common practice, we call this choice of coordinates adapted.

With the background satisfying Eqs. (3.7) and (3.8), the variation (3.6) reduces to the boundary term

$$
\delta_{k} S_{\mathrm{bos}}=\int d \tau\left[\epsilon^{A} \omega_{A \mu} \partial_{\tau} X^{\mu}\right]_{\sigma=0, \pi} .
$$

Using the Dirichlet condition (2.19), this vanishes if and only if, for some scalar field $\alpha_{A}$,

$$
\pi_{\nu}^{\mu} \omega_{A \mu}=\partial_{\nu} \alpha_{A}
$$


on the boundary. Note that this does not imply that $\mathcal{L}_{k_{A}} B_{\mu \nu}=0$, since $\omega_{A}$ may be anything along the Dirichlet directions. More specifically, plugging (3.10) into (3.8) yields

$$
\mathcal{L}_{k_{A}} B_{\mu \nu}=Q_{[\nu, \mu]}^{\lambda} \omega_{A \lambda}+Q_{[\nu}^{\lambda} \partial_{\mu]} \omega_{A \lambda}
$$

If $\epsilon^{A}$ is holomorphic ( $\partial_{=} \epsilon^{A}=0$ only), then the variation (3.6) vanishes up to a boundary term if the Killing vectors satisfy

$$
\nabla_{\mu}^{(+)} k_{A}^{\nu}=0
$$

in the bulk. The remaining boundary term reads

$$
\delta_{k} S_{\mathrm{bos}}=\int d \tau\left[\epsilon^{A} k_{A}^{\mu} E_{\mu \nu} \partial_{\tau} X^{\nu}\right]_{\sigma=0, \pi},
$$

which vanishes if and only if

$$
k_{A}^{\mu} E_{\mu \nu} \pi_{\rho}^{\nu}=0
$$

on the boundary, where we have again used (2.19). In general this may have a solution; however, if the action of $G$ is free and transitive, then there is no solution for this system unless $\pi_{\nu}^{\mu}=0$.

If $\epsilon^{A}$ is antiholomorphic ( $\partial_{+} \epsilon^{A}=0$ only) we similarly find the bulk condition

$$
\nabla_{\mu}^{(-)} k_{A}^{\nu}=0
$$

and the boundary condition

$$
k_{A}^{\mu} E_{\nu \mu} \pi_{\rho}^{\nu}=0
$$

Again, there is no solution when $G$ acts freely and transitively.

Note that (3.12) and (3.14) are satisfied by the chirality conditions on the Killing vectors in the WZW-model [27]. Namely, consider a group manifold of a Lie group $\mathcal{G}$, with isometry group $\mathcal{G} \times \mathcal{G}$ generated by left- and right-invariant Killing vectors $l_{A}^{\mu}$ and $r_{A}^{\mu}$. In the case $\partial_{\underline{\underline{H}}} \epsilon^{A}=0$, the sigma model is invariant under the isometry transformation (3.1) (up to boundary terms and only if (3.7) and (3.8) hold), with $k_{A}^{\mu}$ a linear combination of $l_{A}^{\mu}$ and $r_{A}^{\mu}$. In fact, the model is invariant with respect to both $l_{A}^{\mu}$ and $r_{A}^{\mu}$ separately, and each vector gives rise to an (anti-)chiral current,

$$
\mathcal{J}_{+}^{A} \equiv-r_{\mu}^{A} \partial_{+} X^{\mu}, \quad \mathcal{J}_{-}^{A} \equiv l_{\mu}^{A} \partial_{=} X^{\mu}
$$

The Killing vectors obey the Cartan-Maurer equations,

$$
\nabla_{\rho}^{(+)} r_{A}^{\mu}=0, \quad \nabla_{\rho}^{(-)} l_{A}^{\mu}=0
$$

which are precisely the equations (3.12) and (3.14). 
Finally, if $\epsilon^{A}$ is arbitrary $\left(\partial_{\underline{\underline{*}}} \epsilon^{A} \neq 0\right)$ there is no way to make the variation (3.6) vanish in the bulk, so this case is not allowed.

In conclusion, the bosonic action (3.5) is invariant under the group $G$ if and only if the conditions in Table 2 hold and the parameter $\epsilon^{A}$ is independent of the worldsheet coordinates $\xi^{ \pm}$, i.e., $\partial_{\underline{\underline{\#}}} \epsilon^{A}=0 ; G$ is then an isometry group. Eqs. (2.19) and (3.10) are boundary conditions, whereas Eqs. (3.2), (3.7) and (3.8) hold in the bulk.

$$
\begin{aligned}
Q^{\mu}{ }_{\nu} \partial_{\tau} X^{\nu} & =0 \\
Q^{\mu}{ }_{\nu} k_{A}^{\nu} & =0 \\
\mathcal{L}_{k_{A}} g_{\mu \nu} & =0 \\
\mathcal{L}_{k_{A}} B_{\mu \nu}-\omega_{A[\nu, \mu]} & =0 \\
\pi_{\nu}^{\mu} \omega_{\nu \mu}-\partial_{\nu} \alpha_{A} & =0
\end{aligned}
$$

Table 2: Conditions for the bosonic action (3.5) to be invariant under the group $G$.

\subsubsection{Boundary conditions}

Of the boundary conditions in Table 2 the only one transforming nontrivially under (3.1) is the Dirichlet condition (2.19). Using Eq. (3.2) its variation reads

$$
\epsilon^{A} k_{A}^{\sigma} \pi_{\sigma}^{\rho} \pi_{[\rho, \nu]}^{\mu} \pi_{\lambda}^{\nu} \partial_{\tau} X^{\lambda}=0
$$

implying that

$$
k_{A}^{\sigma} \pi_{\sigma}^{\rho} \pi_{[\rho, \nu]}^{\mu} \pi_{\lambda}^{\nu}=0 .
$$

Thus invariance of the boundary conditions requires the extra condition (3.16).

We also need to check invariance of the boundary relation between left- and right-moving worldsheet fields. The most general classical boundary ansatz compatible with conformal invariance of the bosonic model is

$$
\partial_{=} X^{\mu}=R_{\nu}^{\mu} \partial_{+} X^{\nu}
$$

which is invariant under (3.1) if

$$
\mathcal{L}_{k_{A}} R^{\mu}{ }_{\nu}=0
$$

One can show that this implies

$$
\mathcal{L}_{k_{A}} \pi^{\mu}{ }_{\nu}=\mathcal{L}_{k_{A}} Q^{\mu}{ }_{\nu}=0 .
$$


Thus the conditions for the boundary ansatz (3.17) to be invariant under $G$ are Eqs. (3.18) and (3.19).

\subsubsection{Summary and interpretation}

$$
\begin{aligned}
Q^{\mu}{ }_{\nu} \partial_{\tau} X^{\nu} & =0 \\
Q^{\mu}{ }_{\nu} k_{A}^{\nu} & =0 \\
\mathcal{L}_{k_{A}} g_{\mu \nu} & =0 \\
\mathcal{L}_{k_{A}} B_{\mu \nu}-\partial_{[\mu} \omega_{A \nu]} & =0 \\
\pi_{\mu}^{\nu}{ }_{\mu} \omega_{A \nu}-\partial_{\mu} \alpha_{A} & =0 \\
k_{A}^{\sigma} \pi^{\rho}{ }_{\sigma} \pi^{\mu}{ }_{[\rho, \nu]} \pi^{\nu}{ }_{\lambda} & =0 \\
\partial_{=} X^{\mu}-R_{\nu}^{\mu}{ }_{\nu} \partial_{+} X^{\nu} & =0 \\
\mathcal{L}_{k_{A}} R^{\mu}{ }_{\nu} & =0 \\
\mathcal{L}_{k_{A}} \pi^{\mu}{ }_{\nu}=\mathcal{L}_{k_{A}} Q^{\mu}{ }_{\nu} & =0
\end{aligned}
$$

Table 3: Conditions for invariance of the bosonic nonlinear sigma model under the group $G$.

In Table 3 we summarise the conditions necessary for the bosonic model to be invariant under the group $G$. These conditions may be understood in the following way. First, (2.19) is just the Dirichlet condition, defining the D-brane. Second, (3.2) is the statement that all the generating vectors $k_{A}^{\mu}$ are parallel to the D-brane. We have already seen that the bulk equation (3.7) is the invariance of the metric under the transformation (3.1), implying that $G$ is an isometry group. Together with (3.19) it implies

$$
\mathcal{L}_{k_{A}}\left(\pi_{\rho}^{\mu} g_{\mu \nu} \pi_{\sigma}^{\nu}\right)=0 \text {. }
$$

Moreover, Eq. (3.18) in adapted coordinates means that $R^{\mu}{ }_{\nu}$ is independent of the isometry direction $X^{0}$, and similarly for (3.19). That is, the D-brane is an invariant submanifold under the action of the group $G$ and it is equipped with an invariant metric $\pi_{\rho}^{\mu} g_{\mu \nu} \pi_{\sigma}^{\nu}$.

Next, Eq. (3.8) is a restriction on the background in the bulk which in adapted coordinates states that the dependence of the B-field on the isometry direction is restricted (though not necessarily zero). The condition (3.10) says that the components of $\omega_{A}$ parallel to the brane are restricted, but we cannot say anything about the components orthogonal to it. Eq. (3.17) is just the ansatz we made for the relation between left- and right-movers on the worldsheet.

Finally, we recognise (3.16) as the condition for $\pi_{\nu}^{\mu}$ to be integrable, contracted with $k_{A}^{\mu}$, cf. Eq. (2.18). Thus a restricted form of $\pi$-integrability is a requirement for isometry 
invariance of the bosonic action. This yields a situation geometrically similar to that in [16], implying that the projection of the brane onto the isometry direction in the original model must be an integral submanifold of the target space $M$. Together with (3.8), (3.10) and (3.19), it implies that

$$
k_{A}^{\lambda} \mathcal{L}_{k_{A}}\left(\pi_{\lambda}^{\mu} B_{\mu \nu} \pi_{\rho}^{\nu}\right)=0
$$

i.e., the D-brane is equipped with a B-field $\pi_{\lambda}^{\mu} B_{\mu \nu} \pi_{\rho}^{\nu}$ that is $G$-invariant at least along the isometry directions.

\subsection{Supersymmetric model}

\subsubsection{Action}

The supersymmetric generalisation of the transformation (3.1) reads

$$
\delta_{k} \Phi^{\mu}=\epsilon^{A}(\xi, \theta) k_{A}^{\mu}(\Phi)
$$

where $k_{A}^{\mu}(\Phi)$ is a superfield whose lowest component is $k_{A}^{\mu}(X)$, and $\epsilon^{A}(\xi, \theta)$ is a (superfield) parameter that a priori depends on the worldsheet superspace coordinates $\left(\xi^{ \pm}, \theta^{ \pm}\right)$. The component form of (3.20), which is needed to vary the boundary term in the supersymmetric action (2.1), reads

$$
\left\{\begin{aligned}
\delta_{k} X^{\mu}= & \epsilon^{A}(\xi) k_{A}^{\mu}(X) \\
\delta_{k} \psi_{ \pm}^{\mu}= & \epsilon_{ \pm}^{A}(\xi) k_{A}^{\mu}+\epsilon^{A}(\xi) k_{A, \nu}^{\mu} \psi_{ \pm}^{\nu} \\
\delta_{k} F_{+-}^{\mu}= & \epsilon_{+-}^{A}(\xi) k_{A}^{\mu}+\epsilon_{+}^{A}(\xi) k_{A, \nu}^{\mu} \psi_{-}^{\nu}-\epsilon_{-}^{A}(\xi) k_{A, \nu}^{\mu} \psi_{+}^{\nu} \\
& +\epsilon^{A}(\xi) k_{A, \nu}^{\mu} F_{+-}^{\nu}+\epsilon^{A}(\xi) k_{A, \nu \rho}^{\mu} \psi_{+}^{\rho} \psi_{-}^{\nu}
\end{aligned}\right.
$$

where

$$
\left.\epsilon^{A}(\xi) \equiv \epsilon^{A}(\xi, \theta)\right|_{\theta=0},\left.\quad \epsilon_{ \pm}^{A}(\xi) \equiv\left(D_{ \pm} \epsilon^{A}\right)\right|_{\theta=0},\left.\quad \epsilon_{+-}^{A}(\xi) \equiv\left(D_{+} D_{-} \epsilon^{A}\right)\right|_{\theta=0}
$$

The variation of the action (2.1) under (3.20), (3.21) is given by

$$
\begin{aligned}
\delta_{k} S= & \int d^{2} \xi d^{2} \theta\left[D_{+} \epsilon^{A} D_{-} \Phi^{\nu} E_{\mu \nu} k_{A}^{\mu}+D_{+} \Phi^{\mu} D_{-} \epsilon^{A} E_{\mu \nu} k_{A}^{\nu}+\epsilon^{A} D_{+} \Phi^{\mu} D_{-} \Phi^{\nu} \mathcal{L}_{k_{A}} E_{\mu \nu}\right] \\
& +\frac{i}{2} \int d \tau\left(\epsilon^{A} \mathcal{L}_{k_{A}} B_{\mu \nu}\left[\psi_{+}^{\mu} \psi_{+}^{\nu}+\psi_{-}^{\mu} \psi_{-}^{\nu}\right]+2 k_{A}^{\mu} B_{\mu \nu}\left[\epsilon_{+}^{A} \psi_{+}^{\nu}+\epsilon_{-}^{A} \psi_{-}^{\nu}\right]\right) .
\end{aligned}
$$

If the parameter $\epsilon^{A}$ is constant $\left(D_{ \pm} \epsilon^{A}=0\right)$, then $\delta_{k} S$ vanishes up to boundary terms if the background satisfies (3.7) and (3.8). The remaining boundary terms are

$$
\begin{aligned}
\delta_{k} S= & i \int d^{2} \xi\left[-\partial_{+} D_{-}\left(\epsilon^{A} \omega_{A \mu} D_{-} \Phi^{\mu}\right)+\partial_{=} D_{+}\left(\epsilon^{A} \omega_{A \mu} D_{+} \Phi^{\mu}\right)\right] \\
& +i \int d \tau \epsilon^{A} \partial_{\mu} \omega_{A \nu}\left[\psi_{+}^{\mu} \psi_{+}^{\nu}+\psi_{-}^{\mu} \psi_{-}^{\nu}\right]
\end{aligned}
$$


In components the fermion-terms cancel, and $\delta_{k} S$ reduces to

$$
\delta_{k} S=\int d \tau\left[\epsilon^{A} \omega_{A \mu} \partial_{\tau} X^{\mu}\right]_{\sigma=0, \pi} .
$$

Using the Dirichlet condition (2.19), this vanishes if and only if (3.10) holds on the boundary. Consequently Eq. (3.11) holds, and using $\pi$-integrability (which is provided as a condition for superconformal invariance) it follows that

$$
\pi_{\rho}^{\mu}\left(\mathcal{L}_{k_{A}} B_{\mu \nu}\right) \pi_{\sigma}^{\nu}=0 .
$$

If $\epsilon^{A}$ is (anti-) holomorphic, i.e., $D_{+} \epsilon^{A}=0$ or $D_{-} \epsilon^{A}=0$, then we find the same conditions as in the corresponding bosonic case, see Eqs. (3.12)-(3.15), to which there are no solutions when $G$ acts freely and transitively. If $\epsilon^{A}$ is arbitrary the variation (3.22) does not vanish.

In conclusion, the supersymmetric action (2.1) is invariant under the group $G$ if and only if in addition to the superconformality conditions in Table 1 the conditions listed in Table 4 are satisfied, and if the parameter $\epsilon^{A}$ is independent of the worldsheet supercoordinates $\left(\xi^{ \pm}, \theta^{ \pm}\right)$, i.e., $D_{ \pm} \epsilon^{A}=0$. The group $G$ is then an isometry group, and the action is simultaneously superconformal and isometry invariant.

\begin{tabular}{|rll|}
$Q^{\mu}{ }_{\nu} \partial_{\tau} X^{\nu}$ & $=0$ & $(2.19)$ \\
$Q^{\mu}{ }_{\nu} k_{A}^{\nu}$ & $=0$ & $(3.2)$ \\
$\mathcal{L}_{k_{A}} g_{\mu \nu}$ & $=0$ & $(3.7)$ \\
$\mathcal{L}_{k_{A}} B_{\mu \nu}-\omega_{A[\nu, \mu]}$ & $=0$ & $(3.8)$ \\
$\pi_{\nu}^{\mu} \omega_{A \mu}-\partial_{\nu} \alpha_{A}=0$ & $(3.10)$ \\
$\pi_{\rho}^{\mu}\left(\mathcal{L}_{k_{A}} B_{\mu \nu}\right) \pi^{\nu}{ }_{\sigma}$ & $=0$ & $(3.23)$ \\
\hline
\end{tabular}

Table 4: Conditions for the supersymmetric action (2.1) to be invariant under the group $G$. We have assumed that the conditions listed in Table 1 for $\mathcal{N}=1$ superconformal invariance are satisfied.

\subsubsection{Boundary conditions}

To verify invariance of the boundary conditions in the supersymmetric model we work in 1D superfield formalism (see Appendix B), since this is much more convenient than working in 
components. When $D_{ \pm} \epsilon^{A}=0$ the transformations (3.21) read

$$
\left\{\begin{array}{l}
\delta_{k} X^{\mu}=\epsilon^{A} k_{A}^{\mu}(X) \\
\delta_{k} \psi_{ \pm}^{\mu}=\epsilon^{A} k_{A, \nu}^{\mu} \psi_{ \pm}^{\nu} \\
\delta_{k} F_{+-}^{\mu}=\epsilon^{A} k_{A, \nu}^{\mu} F_{+-}^{\nu}+\epsilon^{A} k_{A, \nu \rho}^{\mu} \psi_{+}^{\rho} \psi_{-}^{\nu}
\end{array}\right.
$$

which in terms of $1 \mathrm{D}$ superfields reads

$$
\left\{\begin{array}{l}
\delta_{k} K^{\mu}=\epsilon^{A} k_{A}^{\mu}(K), \\
\delta_{k} S^{\mu}=\epsilon^{A} k_{A, \nu}^{\mu}(K) S^{\nu},
\end{array}\right.
$$

with $D \epsilon^{A}=0$.

Of the boundary conditions in Tables 1 and 4 the only one transforming nontrivially under $G$ is the Dirichlet condition (2.22). Its variation under (3.24), using Eq. (3.2), reads

$$
k_{A}^{\sigma} \pi_{\sigma}^{\rho} Q_{[\nu, \rho]}^{\mu} \pi_{\lambda}^{\nu} D K^{\lambda}=0
$$

which is satisfied by virtue of $\pi$-integrability, Eq. (2.18).

Next we check invariance of the boundary ansatz (2.21). It is invariant under (3.24) if and only if (3.18) holds, and as a consequence we have also (3.19). Note that in the case of a spacefilling D-brane this is consistent with the condition (3.23) on the background, since then $\mathcal{L}_{k_{A}} B_{\mu \nu}=0$ and from the superconformality condition (2.20) we know that $R^{\mu}{ }_{\nu}=E^{\mu \rho} E_{\nu \rho}$.

\subsubsection{Summary and interpretation}

$\begin{aligned} Q^{\mu}{ }_{\nu} k_{A}^{\nu} & =0 \\ \mathcal{L}_{k_{A}} g_{\mu \nu} & =0\end{aligned}$

Table 5: Conditions for invariance of the $\mathcal{N}=1$ supersymmetric sigma model under the group $G$. We have assumed that the superconformality conditions listed in Table 1 are satisfied.

In Table 5 we summarise the conditions necessary for the supersymmetric sigma model to be invariant under the group $G$, given the superconformality conditions in Table 1 . These 
conditions were all interpreted in Section 3.1.3, except Eq. (3.23). This condition combined with (3.19) implies

$$
\mathcal{L}_{k_{A}}\left(\pi_{\rho}^{\mu} B_{\mu \nu} \pi_{\sigma}^{\nu}\right)=0 \text {. }
$$

Together with the conclusions in Section 3.1.3 and the consequences of the conditions in Table 1, this means the D-brane is a $G$-invariant maximal integral submanifold and it is equipped with invariant geometrical data, namely an invariant metric and an invariant twoform $\left(\pi_{\rho}^{\mu} g_{\mu \nu} \pi_{\sigma}^{\nu}\right.$ and $\pi_{\rho}^{\mu} B_{\mu \nu} \pi_{\sigma}^{\nu}$, respectively).

\section{Bosonic T-duality}

Before studying the supersymmetric sigma model, we first highlight some fundamental issues concerning gauging and T-duality that arise already in the bosonic model. Most aspects of T-duality are well understood in the absence of a boundary $[2,3,11,12]$. Here we use the gauging procedure [6] to obtain the dual model via a parent action with boundaries, determining the boundary conditions necessary for this procedure to work. This analysis involves several checks. First, the act of integrating out fields to obtain the original and dual sigma models must be consistent, meaning that the parent action must be gauge invariant through every step before the gauge is fixed. Second, the boundary equations of motion must be gauge invariant as well as mutually compatible. Third, the boundary conditions themselves must be gauge invariant [28]. After completing these checks, we next find the most general ansatz for the relation between left- and right-movers and derive the conditions for this ansatz to be gauge invariant.

We consider the simplest possible case, i.e., we gauge a $U(1)$ isometry generated by a single Killing vector $k^{\mu}$.

\subsection{Consistent T-duality procedure}

Gauging the $U(1)$ isometry in the bosonic action (3.5) produces the following parent action,

$$
\begin{aligned}
S_{P}= & \int d^{2} \xi\left[\left(\partial_{+} X^{\mu}+k^{\mu} A_{+}\right)\left(\partial_{=} X^{\nu}+k^{\nu} A_{=}\right) E_{\mu \nu}\right. \\
& \left.+A_{=} \partial_{+} y-A_{+} \partial_{=} y+A_{=} \omega_{\mu} \partial_{+} X^{\mu}-A_{+} \omega_{\mu} \partial_{=} X^{\mu}\right] .
\end{aligned}
$$

Here we have introduced independent gauge fields $A_{\#}$ and a Lagrange multiplier $y$. If the background satisfies (3.7) and (3.8), then ${ }^{7} S_{P}$ is invariant up to boundary terms under the

\footnotetext{
${ }^{7}$ Choosing a gauge for $\omega$ such that $\mathcal{L}_{k} B_{\mu \nu}=\omega_{[\nu, \mu]}=0$ is possible only when there is just one Killing vector $k^{\mu}$. If the isometry group were, say, $U(1) \times U(1)$, with two commuting Killing vectors $k_{1}^{\mu}, k_{2}^{\mu}$, then it
} 
following gauge transformations,

$$
\left\{\begin{array}{l}
\delta_{k} X^{\mu}=\epsilon(\xi) k^{\mu}(X) \\
\delta_{k} A_{\underline{\underline{\#}}}=-\partial_{\underline{\underline{\underline{H}}}} \epsilon(\xi) \\
\delta_{k} y^{=}-\epsilon(\xi) k^{\mu}(X) \omega_{\mu}(X)
\end{array}\right.
$$

where the gauge parameter $\epsilon$ is an unrestricted function of the worldsheet coordinates. We can rewrite $S_{P}$ in terms of gauge covariant quantities, i.e., we make the substitution ${ }^{8}$

$$
\nabla_{\underline{\underline{H}}} X^{\mu} \equiv \partial_{\underline{\underline{\#}}} X^{\mu}+k^{\mu} A_{\underline{\underline{\#}}}
$$

which transforms covariantly under (4.2),

$$
\delta_{k} \nabla_{\underline{\underline{\#}}} X^{\mu}=\epsilon k_{, \nu}^{\mu} \nabla_{\underline{\underline{H}}} X^{\nu}
$$

Then the parent action reads

$$
\begin{aligned}
S_{P}= & \int d^{2} \xi\left[\nabla_{+} X^{\mu} \nabla_{=} X^{\nu} E_{\mu \nu}\right. \\
& \left.+A_{=} \partial_{+} y-A_{+} \partial_{=} y+A_{=} \omega_{\mu} \nabla_{+} X^{\mu}-A_{+} \omega_{\mu} \nabla_{=} X^{\mu}\right]
\end{aligned}
$$

This substitution will be useful in Section 4.5 when discussing a boundary ansatz for the worldsheet fields.

\subsubsection{Equations of motion}

From the parent action the original and dual sigma models are obtained by integrating out the fields $y$ and $A_{\underline{\underline{\#}}}$, respectively, and then fixing the gauge. To integrate out the fields, we need their equations of motion, both in the bulk and on the boundary. The bulk equation of motion resulting from a variation of $y$ is

$$
\partial_{+} A_{=}-\partial_{=} A_{+}=0
$$

implying that

$$
A_{\underline{\underline{\#}}}=\partial_{\underline{\underline{\#}}} \lambda
$$

for some scalar field $\lambda$. From varying $A_{\underline{\underline{\#}}}$ we find the bulk equations

$$
\left\{\begin{array}{l}
A_{+} k^{\mu} k^{\nu} E_{\mu \nu}+\partial_{+} X^{\nu}\left(k^{\mu} E_{\nu \mu}+\omega_{\nu}\right)+\partial_{+} y=0 \\
A_{=} k^{\mu} k^{\nu} E_{\mu \nu}+\partial_{=} X^{\nu}\left(k^{\mu} E_{\mu \nu}-\omega_{\nu}\right)-\partial_{=} y=0
\end{array}\right.
$$

would not be possible to gauge away both Lie derivatives $\mathcal{L}_{k_{1,2}} B_{\mu \nu}$ simultaneously; see e.g. [11, 29]. This is true for models without boundary.

${ }^{8}$ These $\nabla_{\underline{\underline{\#}}}$ operators have nothing to do with the $\nabla_{ \pm}^{( \pm)}$operators defined in $(2.5)$. 
In addition, the variation with respect to $y$ produces a boundary term (there are no boundary terms from varying $A_{\underline{\underline{\underline{H}}}}$,

$$
\int d \tau \delta y\left(A_{+}+A_{=}\right)=0
$$

To find the most general solution to (4.7) that is compatible with the bulk equation of motion (4.5), we restrict the latter to the boundary and insert it in (4.7). The resulting condition reads

$$
2 \int d \tau \delta y \partial_{\tau} \lambda=0
$$

which has two solutions: $\partial_{\tau} y=0$ and $\partial_{\tau} \lambda=0$. The latter solution, however, is not allowed, since it would entail a restriction of the gauge freedom of the action. After integrating out the fields, but before fixing the gauge, the action must still be gauge invariant, otherwise we will not be able to use the gauge transformations (4.2) to choose a gauge and perform T-duality. Thus we are left with

$$
\partial_{\tau} y=0
$$

as the most general solution to (4.7).

\subsubsection{Recovering the original model}

We may now proceed to integrate out $y$ by substituting (4.5) and (4.9) in the parent action (4.3). Having thus eliminated $y$ both in the bulk and on the boundary, the resulting action reads

$$
\begin{aligned}
S_{\lambda}= & \int d^{2} \xi\left[\left(\partial_{+} X^{\mu}+k^{\mu} \partial_{+} \lambda\right)\left(\partial_{=} X^{\nu}+k^{\nu} \partial_{=} \lambda\right) E_{\mu \nu}\right. \\
& \left.+\partial_{=} \lambda \omega_{\mu} \partial_{+} X^{\mu}-\partial_{+} \lambda \omega_{\mu} \partial_{=} X^{\mu}\right] .
\end{aligned}
$$

Before fixing the gauge we need to check that $S_{\lambda}$ is gauge invariant. It turns out that this requirement yields another crucial boundary condition, which must be satisfied if the T-duality operation is to be well-defined. Given (3.7) and (3.8), the transformation of $S_{\lambda}$ under (4.2) yields the following boundary term,

$$
\delta_{k} S_{\lambda}=2 \int d \tau \epsilon \omega_{\mu}\left(\partial_{\tau} X^{\mu}+k^{\mu} \partial_{\tau} \lambda\right)
$$

There are two possibilities for making this term vanish. One is to require that the gauge parameter $\epsilon$ be zero on the boundary,

$$
\left.\epsilon\right|_{\sigma=0, \pi}=0
$$

However, this would restrict the gauge freedom of our action, which, as remarked above, is not allowed. Thus the only permissible condition that sets (4.11) to zero is

$$
\omega_{\mu}\left(\partial_{\tau} X^{\mu}+k^{\mu} \partial_{\tau} \lambda\right)=0
$$


Using the Dirichlet condition $Q_{\nu}^{\mu} \partial_{\tau} X^{\nu}=0$, and since $\pi_{\nu}^{\mu} \partial_{\tau} X^{\nu}$ is in general nonzero, the condition (4.13) is equivalent to

$$
\pi_{\nu}^{\mu} \omega_{\mu}=0
$$

That is, the Neumann components of $\omega_{\mu}$ vanish on the boundary. Note that, since $Q^{\mu}{ }_{\nu} k^{\nu}=0$, Eq. (3.2), this implies $k^{\mu} \omega_{\mu}=0$, i.e., $\omega_{\mu}$ is orthogonal to the Killing vector.

We conclude that the action (4.10) is gauge invariant given the two boundary conditions (4.9) and (4.14), and we can now safely proceed with the gauge-fixing. We go to adapted coordinates $\left(k^{\mu}=\delta_{0}^{\mu}\right)$ and choose a gauge where $\lambda=0$. The action $S_{\lambda}$ then reduces to the original nonlinear sigma model,

$$
S_{\mathrm{bos}}=\int d^{2} \xi \partial_{+} X^{\mu} \partial_{=} X^{\nu} E_{\mu \nu} .
$$

\subsubsection{Finding the dual model}

To find the dual model we use (4.6) to integrate out $A_{\underline{\underline{\#}}}$ in (4.3). The resulting action reads

$$
\begin{aligned}
S_{y}= & \int d^{2} \xi\left[\partial_{+} X^{\mu} \partial_{=} X^{\nu} E_{\mu \nu}+\left(k^{\lambda} k^{\sigma} E_{\lambda \sigma}\right)^{-1} \times\right. \\
& \left.\times\left(\partial_{+} y+\omega_{\mu} \partial_{+} X^{\mu}+k^{\mu} E_{\nu \mu} \partial_{+} X^{\nu}\right)\left(\partial_{=} y+\omega_{\rho} \partial_{=} X^{\rho}-k^{\gamma} E_{\gamma \rho} \partial_{=} X^{\rho}\right)\right],
\end{aligned}
$$

which is required to be gauge invariant. Using (3.7) and (3.8) a gauge transformation leaves this action invariant up to the following boundary term,

$$
\delta_{k} S_{y}=2 \int d \tau \epsilon\left(\partial_{\tau} y+\omega_{\mu} \partial_{\tau} X^{\mu}\right) .
$$

But we already know from Sections 4.1.1 and 4.1.2 that (4.9) and (4.14) must always hold. Hence (4.17) vanishes identically, and $S_{y}$ is indeed gauge invariant. We can therefore go to adapted coordinates and choose the gauge where $X^{0}=0$. This yields the action

$$
\widetilde{S}_{\text {bos }}=\int d^{2} \xi \partial_{+} \widetilde{X}^{\mu} \partial_{=} \widetilde{X}^{\nu} \widetilde{E}_{\mu \nu}
$$

where

$$
\widetilde{X}^{0} \equiv y, \quad \widetilde{X}^{n} \equiv X^{n},
$$

and $\widetilde{E}_{\mu \nu}$ is the dual background given by the following (modified) Buscher's rules [4, 5, 6],

$$
\left\{\begin{array}{l}
\widetilde{E}_{00}=E_{00}^{-1}, \\
\widetilde{E}_{0 n}=-\left(E_{0 n}-\omega_{n}\right) E_{00}^{-1}, \\
\widetilde{E}_{m 0}=\left(E_{m 0}+\omega_{m}\right) E_{00}^{-1}, \\
\widetilde{E}_{m n}=E_{m n}-\left(E_{m 0}+\omega_{m}\right)\left(E_{0 n}-\omega_{n}\right) E_{00}^{-1} .
\end{array}\right.
$$

Thus we have found the dual action, Eq. (4.18), which has the same form as the original one, with $y$ playing the part of $X^{0}$-coordinate. 


\subsubsection{Summary}

In Table 6 we collect the boundary conditions necessary for the bosonic parent action (4.3) to give rise to the original and dual nonlinear sigma models in a consistent manner.

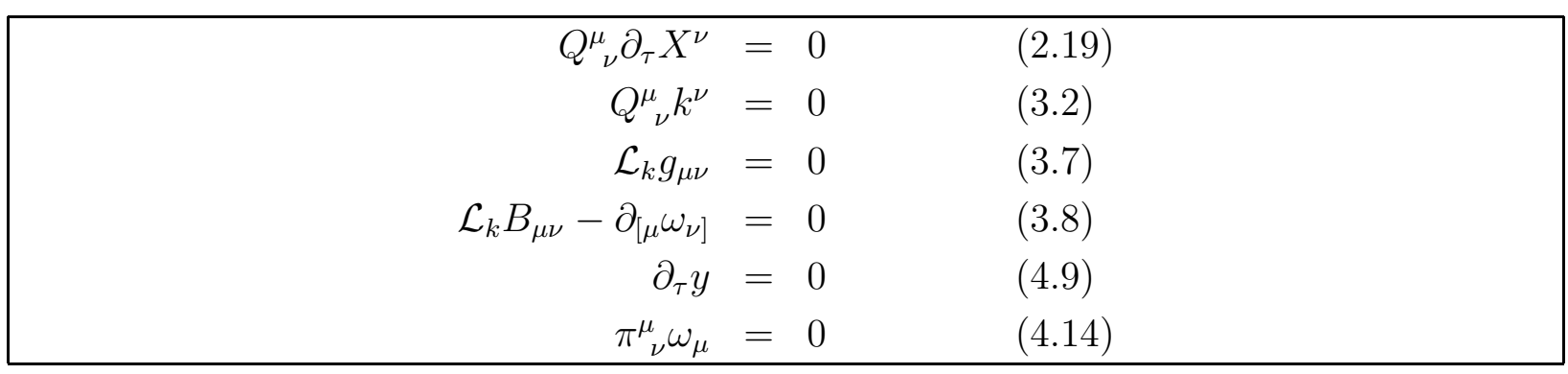

Table 6: Conditions for the bosonic parent action (4.3) to consistently yield the original and dual nonlinear sigma models.

\subsection{Gauge invariance of parent action}

We know that the parent action is gauge invariant on the boundary as well as in the bulk after integrating out the lagrange multiplier $y$ and gauge fields $A_{\underline{\underline{H}}}$, respectively, given the conditions in Table 6 . Let us nevertheless make sure that the parent action is gauge invariant also before the fields are integrated out. This is quickly done: a gauge transformation of the parent action (4.3) leaves precisely the boundary term (4.17), which vanishes identically due to $(2.19)$, (4.9) and (4.14).

\subsection{Compatibility of field equations}

Here we check that the equations of motion derived from the parent action are consistent on the boundary. This means checking that all boundary field equations are mutually compatible as well as compatible with the restriction of bulk equations to the boundary. We already have the equations of motion for $y$ and $A_{\#}$, so let us derive the one remaining boundary field equation, namely that of $X^{\mu}$. A field variation with respect to $X^{\mu}$ produces the following equation,

$$
\int d \tau \delta X^{\mu}\left[\left(\partial_{=} X^{\nu}+k^{\nu} A_{=}\right) E_{\mu \nu}-\left(\partial_{+} X^{\nu}+k^{\nu} A_{+}\right) E_{\nu \mu}+\left(A_{+}+A_{=}\right) \omega_{\mu}\right]=0
$$


implying

$$
\pi_{\rho}^{\mu}\left[\left(\partial_{=} X^{\nu}+k^{\nu} A_{=}\right) E_{\mu \nu}-\left(\partial_{+} X^{\nu}+k^{\nu} A_{+}\right) E_{\nu \mu}\right]+\pi_{\rho}^{\mu} \omega_{\mu}\left(A_{+}+A_{=}\right)=0 .
$$

This is compatible with restricting the bulk equations of motion (4.4) and (the difference between) Eqs. (4.6) to the boundary if and only if the following boundary condition holds,

$$
\partial_{\tau} y+\omega_{\mu} \pi_{\nu}^{\mu}\left(\partial_{\tau} X^{\nu}+k^{\nu} \partial_{\tau} \lambda\right)=0
$$

Inserting (4.9) and (4.14), the left-hand side vanishes identically, showing that the $X^{\mu}$ boundary equation of motion is compatible with the $y$ boundary equation of motion and the bulk equations of motion for $A_{+}$and $y$. Hence the parent action (4.3) is consistent at the level of field equations, given the $\overline{\bar{b}}$ oundary conditions in Table 6.

\subsection{Gauge invariance of boundary conditions}

For complete consistency, we must also check that all the boundary conditions we have used in this section are themselves gauge invariant. We list them here:

$$
\begin{aligned}
Q_{\nu}^{\mu} \partial_{\tau} X^{\nu} & =0 \\
Q^{\mu}{ }_{\nu} k^{\nu} & =0 \\
\mathcal{L}_{k} g_{\mu \nu} & =0 \\
\mathcal{L}_{k} B_{\mu \nu}-\partial_{[\mu} \omega_{\nu]} & =0 \\
\partial_{+} A_{=}-\partial_{=} A_{+} & =0 \\
A_{+} k^{\mu} k^{\nu} E_{\mu \nu}+\partial_{+} X^{\nu}\left(k^{\mu} E_{\nu \mu}+\omega_{\nu}\right)+\partial_{+} y & =0 \\
A_{=} k^{\mu} k^{\nu} E_{\mu \nu}+\partial_{=} X^{\nu}\left(k^{\mu} E_{\mu \nu}-\omega_{\nu}\right)-\partial_{=} y & =0 \\
\partial_{\tau} y & =0 \\
\pi_{\nu}^{\mu} \omega_{\mu} & =0 \\
\pi_{\rho}^{\mu}\left[\left(\partial_{=} X^{\nu}+k^{\nu} A_{=}\right) E_{\mu \nu}-\left(\partial_{+} X^{\nu}+k^{\nu} A_{+}\right) E_{\nu \mu}\right]+\pi_{\rho}^{\mu} \omega_{\mu}\left(A_{+}+A_{=}\right) & =0
\end{aligned}
$$

The requirements for gauge invariance of each of these conditions are as follows:

1. Eqs. (3.2), (3.7), (3.8), (4.4) and (4.14) are trivially gauge invariant.

2. (2.19) is gauge invariant if (3.16) holds.

3. Eqs. (4.6) are gauge invariant due to (3.8).

4. (4.9) is gauge invariant if $k^{\mu} \omega_{\mu}=0$, which follows from (4.14).

5. Given (4.14), (4.21) is gauge invariant if $Q^{\rho}{ }_{\mu} \mathcal{L}_{k} \pi^{\mu}{ }_{\nu}=0$, which is equivalent to Eq. (3.16).

In conclusion, our boundary conditions are gauge invariant provided (3.16) holds. 


\subsection{Boundary ansatz}

We now turn to the ansatz for the relation between left- and right-movers on the boundary. This is needed for conformal invariance of the gauged model, and provides us with boundary conditions in terms of a gluing matrix. In the bosonic ungauged model, the most general ansatz reads

$$
\partial_{=} X^{\mu}=R_{\nu}^{\mu} \partial_{+} X^{\nu}
$$

In the gauged model, however, it turns out that the appropriate ansatz is

$$
\nabla_{=} X^{\mu}=R_{\nu}^{\mu} \nabla_{+} X^{\nu}
$$

This is supported by an analysis of the conformal current (the stress-energy tensor) of the gauged model. For the model to be conformal on the boundary, the left- and right-moving components $T_{ \pm \pm}$must satisfy

$$
0=T_{++}-T_{--} \equiv \nabla_{+} X^{\mu} g_{\mu \nu} \nabla_{+} X^{\nu}+\nabla_{=} X^{\mu} g_{\mu \nu} \nabla_{=} X^{\nu}
$$

The most general solution to this is obtained by inserting (4.23), which leads to the condition (2.11), i.e., $R_{\nu}^{\mu}$ must preserve the metric, the same condition required for the ungauged model to be conformally invariant. If we were to use (3.17) instead, we would in addition to (2.11) find additional, very restrictive conditions, hence that solution would be less general.

What other boundary conditions, in addition to (2.11), follow from the ansatz (4.23)? Gauge invariance of (4.23) requires that $\mathcal{L}_{k} R^{\mu}{ }_{\nu}=0$, Eq. (3.18), which implies $\mathcal{L}_{k} \pi^{\mu}{ }_{\nu}=\mathcal{L}_{k} Q^{\mu}{ }_{\nu}=$ 0, Eq. (3.19). Two further conditions are obtained by inserting (4.23) into the equation of motion (4.21), and using $\pi_{\nu}^{\mu} \omega_{\mu}=0$, Eq. (4.14). The result is Eq. (2.17) (diagonalisation of the metric) and Eq. (2.20), which arose in the analysis of the ungauged supersymmetric model in $[16,26]$, but here they arise as conformality conditions in the gauged bosonic model.

One may ask if the gluing matrix $R^{\mu}{ }_{\nu}$ of the gauged model is the same object as the gluing matrix in the ungauged model, something that is not a priori clear. We can see that in fact this must be the case, by the following simple observation. The current associated to conformal symmetry of the gauged model has a form identical to that of the original model, with the original fields replaced by gauge covariant ones. As a consequence the analysis of conformal invariance of the parent action is identical to that of the ungauged action. Hence the resulting conditions for the gluing matrix of the gauged model have the same form as those of the original model. These conditions should reduce to those of the original model when going to adapted coordinates and fixing the gauge. The question is then whether there is a generalisation of the gluing matrix, depending on more fields than $X^{\mu}$, which reduces to $R_{\nu}^{\mu}$ once we integrate out $y$ and set $k^{\mu}=\delta_{0}^{\mu}$ and $A_{\underline{\underline{\#}}}=0$. The answer is no: for conformal 
reasons, the only field the gluing matrix can depend on other than $X^{\mu}$ is $y$, but there is no conformally allowed modification of $R^{\mu}{ }_{\nu}$ that vanishes when we use the $y$ field equations and fix the gauge. On the other hand, the ansatz (4.23) reduces in a trivial way to the ansatz (3.17) of the original model.

\subsection{Summary and interpretation}

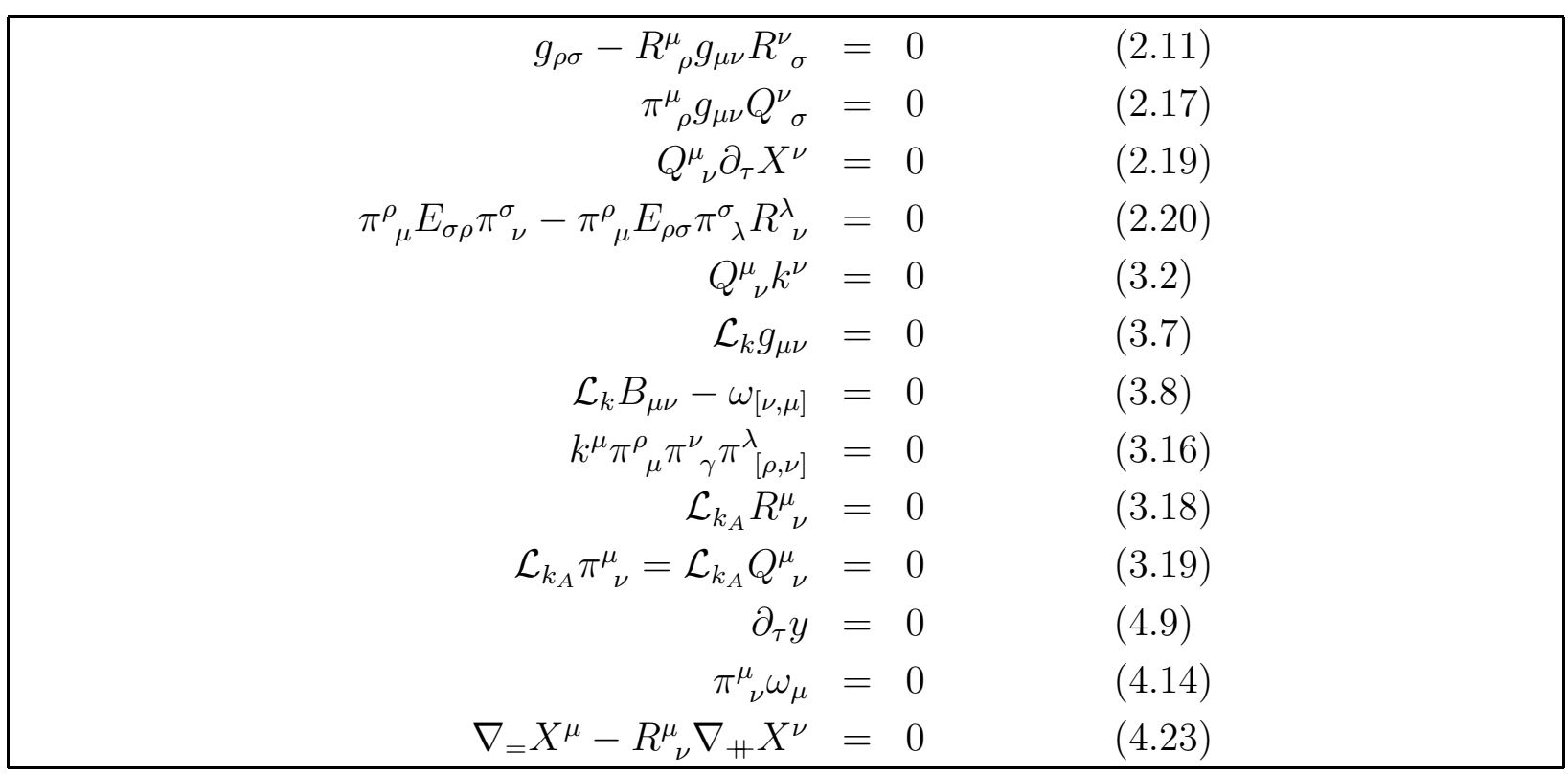

Table 7: Conditions for the bosonic nonlinear sigma model to be consistent under T-duality.

In Table 7 we summarise the boundary conditions necessary for the bosonic nonlinear sigma model to be consistent under T-duality. The first four of these conditions were found and interpreted already in Section 2: (2.11) is the preservation of the metric, (2.17) is the diagonalisation of the metric, (2.19) is the Dirichlet condition in the original model, and (2.20) implies indirectly that the B-field on the D-brane is the pullback of the background B-field.

Conditions (3.2), (3.7), (3.8), (3.16), (3.18) and (3.19) were interpreted already in Section 3.1.3. Together with (4.14) these conditions tell us what we already knew about the D-brane in the original model, namely that it is an isometry invariant submanifold equipped with an invariant metric and two-form, whose projection onto the isometry direction is an integral submanifold. 
Next, Eq. (4.9) is a Dirichlet condition for $y$, implying that the brane on the dual side is transverse to the $y$-direction. This is consistent with the expectation that T-duality parallel to a D-brane decreases its dimension by one. The condition (4.14) means that $\omega_{\mu}$ has no components parallel to the brane in the original sigma model, but we cannot say anything about the components orthogonal to it. Eq. (4.23) is just the ansatz we made for the relation between left- and right-movers on the worldsheet.

\section{$5 \quad$ Supersymmetric T-duality}

The bosonic discussion generalises in most respects straightforwardly to the supersymmetric model. We follow the same logic, starting with consistency of the T-duality procedure, then checking gauge invariance and compatibility of field equations. Here, however, we find that a boundary ansatz for the worldsheet fields is needed at an early stage to finish the analysis of the field equations. We therefore make a brief digression at the appropriate time to discuss the ansatz, and then complete the consistency check. In addition, we check gauge invariance of the boundary conditions and verify that the action as well as all boundary conditions are compatible with supersymmetry.

\subsection{Consistency of T-duality procedure}

Gauging the $U(1)$ isometry in the bulk of the supersymmetric action (2.1) produces the following parent action,

$$
\begin{aligned}
S_{P}= & \int d^{2} \xi d^{2} \theta\left[\nabla_{+} \Phi^{\mu} \nabla_{-} \Phi^{\nu} E_{\mu \nu}(\Phi)+\omega_{\mu} D_{(+} \Phi^{\mu} V_{-)}+D_{(-} Y V_{+)}\right] \\
& +\frac{i}{2} \int d \tau B_{\mu \nu}\left[\psi_{+}^{\mu} \psi_{+}^{\nu}+\psi_{-}^{\mu} \psi_{-}^{\nu}\right],
\end{aligned}
$$

where $^{9}$

$$
\nabla_{ \pm} \Phi^{\mu} \equiv D_{ \pm} \Phi^{\mu}+k^{\mu} V_{ \pm}
$$

and the independent superfields $V_{ \pm}$and $Y$ are, respectively, gauge fields and a Lagrange multiplier. Given (3.7) and (3.8), $S_{P}$ is invariant up to boundary terms under the following gauge transformations,

$$
\left\{\begin{aligned}
\delta_{k} \Phi^{\mu} & =\epsilon(\xi, \theta) k^{\mu}(\Phi), \\
\delta_{k} V_{ \pm} & =-D_{ \pm} \epsilon(\xi, \theta), \\
\delta_{k} Y & =-\epsilon(\xi, \theta) k^{\mu}(\Phi) \omega_{\mu}(\Phi) .
\end{aligned}\right.
$$

\footnotetext{
${ }^{9}$ These $\nabla_{ \pm}$operators are unrelated to the $\nabla_{ \pm}^{( \pm)}$operators defined in (2.5).
} 
One may now ask if not the boundary term in (5.1) should also be modified in some way, to make the full action gauge invariant and consistent also on the boundary. First we note that leaving the boundary term unchanged leads to very restrictive conditions on the background, e.g., $k^{\mu} B_{\mu \nu} \pi_{\rho}^{\nu}=0$, implying that a modification is indeed necessary. However, it is not immediately clear what this modification should look like. We make the natural choice to replace the worldsheet fermions $\psi_{ \pm}^{\mu}$ with their gauge covariant counterparts, defined by

$$
\left.\widehat{\psi}_{ \pm}^{\mu} \equiv \nabla_{ \pm} \Phi^{\mu}\right|_{\theta=0}=\psi_{ \pm}^{\mu}+k^{\mu} v_{ \pm}
$$

where

$$
\left.v_{ \pm} \equiv V_{ \pm}\right|_{\theta=0}
$$

With this modification of the boundary term, the gauged action now reads

$$
\begin{aligned}
\widehat{S}= & \int d^{2} \xi d^{2} \theta\left[\nabla_{+} \Phi^{\mu} \nabla_{-} \Phi^{\nu} E_{\mu \nu}(\Phi)+\omega_{\mu} D_{(+} \Phi^{\mu} V_{-)}+D_{(-} Y V_{+)}\right] \\
& +\frac{i}{2} \int d \tau B_{\mu \nu}\left[\widehat{\psi}_{+}^{\mu} \widehat{\psi}_{+}^{\nu}+\widehat{\psi}_{-}^{\mu} \widehat{\psi}_{-}^{\nu}\right] .
\end{aligned}
$$

This is the action we will work with, and we will see that it is consistent and supersymmetric. We begin by showing that it gives rise to the appropriate original and dual sigma models in a consistent way.

\subsubsection{Equations of motion}

To obtain the original and dual models we need to integrate out $Y$ and $V_{ \pm}$, respectively, by using their field equations in (5.3), and then fix the gauge. A variation with respect to $Y$ yields the following bulk equation of motion,

$$
D_{+} V_{-}+D_{-} V_{+}=0
$$

implying

$$
V_{ \pm}=D_{ \pm} \Lambda
$$

for some scalar superfield $\Lambda$. Varying $V_{ \pm}$yields the field equations

$$
\left\{\begin{array}{l}
V_{+} k^{\mu} k^{\nu} E_{\mu \nu}+D_{+} \Phi^{\nu}\left(k^{\mu} E_{\nu \mu}+\omega_{\nu}\right)+D_{+} Y=0 \\
V_{-} k^{\mu} k^{\nu} E_{\mu \nu}+D_{-} \Phi^{\nu}\left(k^{\mu} E_{\mu \nu}-\omega_{\nu}\right)-D_{-} Y=0 .
\end{array}\right.
$$

The boundary term from varying $Y$ reads $^{10}$

$$
\int d \tau\left[-\left(v_{++}+v_{--}\right) \delta y+v_{+} \delta y_{+}+v_{-} \delta y_{-}\right]=0
$$

\footnotetext{
${ }^{10}$ Note that there are no boundary terms arising from the variation with respect to $V_{ \pm}$, nor are there any $v_{ \pm}$-terms in the boundary term that might contribute, since we are using the covariant variables $\widehat{\psi}_{ \pm}^{\mu}$ rather than the combination $\psi_{ \pm}^{\mu}+k^{\mu} v_{ \pm}$.
} 
where

$$
\left.v_{ \pm \pm} \equiv\left(D_{ \pm} V_{ \pm}\right)\right|_{\theta=0},\left.\quad y_{ \pm} \equiv\left(D_{ \pm} Y\right)\right|_{\theta=0},\left.\quad y \equiv Y\right|_{\theta=0}
$$

In the interest of generality, we need to be careful when solving (5.7). Since $Y$ is a Lagrange multiplier, so we know that it is definitely independent of all other fields, it is safest - and easiest - to first analyse the components of this field rather than those of $V_{ \pm}$. More precisely, we need to find the most general boundary condition relating $y_{+}$to $y_{-}$, and then substitute this relation in (5.7) before solving it. Dimensional considerations (classical conformality), analogous to those that led to the most general linear fermionic ansatz in the original model, suggest that the ansatz for $y_{ \pm}$must take the form

$$
y_{-}+a y_{+}=0
$$

where $a$ is some scalar field. Because $Y$ is independent of the other fields, $a$ must also be independent of them. However, a may a priori depend on $y$. We can determine whether or not this is the case by examining the superpartner of (5.8), which reads

$$
a \partial_{+} y+\eta \partial_{=} y=(\eta a-1) y_{+-}-\partial_{y} a\left(y_{+}+\eta y_{-}\right) y_{+}
$$

Due to (5.8), the last term on the right-hand side is annihilated by $y_{+}$, and we have

$$
a \partial_{+} y+\eta \partial_{=} y=(\eta a-1) y_{+-} .
$$

On the other hand we know from Section 4.1 that (5.9) should reduce to $\partial_{\tau} y=0$ in the bosonic case. Since in the bosonic model $y_{+-}=0$, this is true if and only if $a=\eta$. Hence the most general ansatz for $y_{ \pm}$is

$$
y_{-}+\eta y_{+}=0
$$

Returning to the equation of motion (5.7), the insertion of (5.10) reduces it to

$$
-\left(v_{++}+v_{--}\right) \delta y+\left(v_{+}-\eta v_{-}\right) \delta y_{+}=0 .
$$

The two terms must vanish independently. ${ }^{11}$ From the first term it is clear that $\delta y=0$, because $v_{++}+v_{--}$is not allowed to vanish, for the same reason (gauge freedom) that $A_{+}+A_{=}$had to be nonzero in Section 4.1. More precisely, the gauge transformation of $v_{++}+v_{--}=0$ is $-2 i \partial_{\tau} \epsilon=0$, which would restrict the gauge. We thus find

$$
\partial_{\tau} y=0
$$

The second term in (5.11) allows two possibilities:

$$
v_{-}-\eta v_{+}=0
$$

\footnotetext{
${ }^{11}$ There were at the outset the three independent fields $\delta y, \delta y_{ \pm}$, and we have used (5.10) to eliminate $\delta y_{-}$, leaving the two independent fields $\delta y, \delta y_{+}$.
} 
or

$$
\delta y_{+}=0 .
$$

However, it turns out that the latter condition is not a good solution, because it does not eliminate $y_{+}$on the boundary when used in the parent action (which was the whole point of using the $Y$ field equations in the action). To see this, consider the boundary $Y$-terms remaining after substituting the bulk equations of motion (5.5) in (5.3),

$$
\int d \tau\left[-2 \lambda \partial_{\tau} y+i\left(v_{+}-\eta v_{-}\right) y_{+}\right]
$$

where $\left.\lambda \equiv \Lambda\right|_{\theta=0}$. The first term vanishes due to (4.9), and (5.12) cancels the second term, whereas (5.13) would not cancel it. Hence we must adopt (5.12), and this is the boundary equation of motion for $y_{+}$.

In Table 8 we summarise the boundary conditions implied by $Y$-on-shell analysis. Note that (4.9) and (5.10) are off-shell conditions imposed by hand, whereas (5.12) a priori holds only on-shell. However, we will see presently that in fact this condition also must be off-shell.

$$
\begin{aligned}
\partial_{\tau} y & =0 \\
y_{-}+\eta y_{+} & =0 \\
v_{-}-\eta v_{+} & =0
\end{aligned}
$$

Table 8: Conditions found when integrating out the $Y$-field in the supersymmetric parent action (5.3).

\subsubsection{Recovering the original model}

Having derived the most general field equations for $Y$, we now use them to integrate out $Y$ in the parent action (5.3). Thus, using Eq. (5.5) and the conditions in Table 8, the result is

$$
\begin{aligned}
S_{\Lambda}= & \int d^{2} \xi d^{2} \theta\left[\left(D_{+} \Phi^{\mu}+k^{\mu} D_{+} \Lambda\right)\left(D_{-} \Phi^{\nu}+k^{\nu} D_{-} \Lambda\right) E_{\mu \nu}+\omega_{\mu} D_{(+} \Phi^{\mu} D_{-)} \Lambda\right] \\
& +\frac{i}{2} \int d \tau B_{\mu \nu}\left[\widehat{\psi}_{+}^{\mu} \widehat{\psi}_{+}^{\nu}+\widehat{\psi}_{-}^{\mu} \widehat{\psi}_{-}^{\nu}\right] .
\end{aligned}
$$

This action must be gauge invariant. Its gauge transformation, using (3.7) and (3.8), is a pure boundary term,

$$
\begin{aligned}
\delta_{k} S_{\Lambda}= & \int d \tau\left[2 \epsilon \omega_{\mu}\left(\partial_{\tau} X^{\mu}+k^{\mu} \partial_{\tau} \lambda\right)+i \epsilon\left(\omega_{\nu, \mu} k^{\mu}+\omega_{\mu} k_{, \nu}^{\mu}\right)\left(\lambda_{+} \widehat{\psi}_{+}^{\nu}+\lambda_{-} \widehat{\psi}_{-}^{\nu}\right)\right. \\
& \left.-i \epsilon_{+} \omega_{\mu} \widehat{\psi}_{+}^{\mu}-i \epsilon_{-} \omega_{\mu} \widehat{\psi}_{-}^{\mu}\right]
\end{aligned}
$$


where

$$
\left.\epsilon_{ \pm} \equiv\left(D_{ \pm} \epsilon\right)\right|_{\theta=0},\left.\quad \lambda_{ \pm} \equiv\left(D_{ \pm} \Lambda\right)\right|_{\theta=0} .
$$

Note that the first term in (5.15) is the expected bosonic part of the variation, cf. Eq. (4.11). To find the conditions necessary for $\delta_{k} S_{\Lambda}$ to vanish we make use of the fact that gauge invariance of (5.12) yields a relation between the gauge parameters $\epsilon_{ \pm}$,

$$
\epsilon_{-}-\eta \epsilon_{+}=0 .
$$

In addition, (5.12) implies $\lambda_{-}-\eta \lambda_{+}=0$, which we insert together with (5.16) in (5.15) to get

$$
\begin{aligned}
\delta_{k} S_{\Lambda}= & \int d \tau\left[2 \epsilon \omega_{\mu}\left(\partial_{\tau} X^{\mu}+k^{\mu} \partial_{\tau} \lambda\right)+i \epsilon\left(\omega_{\nu, \mu} k^{\mu}+\omega_{\mu} k_{, \nu}^{\mu}\right) v_{+}\left(\widehat{\psi}_{+}^{\nu}+\eta \widehat{\psi}_{-}^{\nu}\right)\right. \\
& \left.-i \epsilon_{+} \omega_{\mu}\left(\widehat{\psi}_{+}^{\mu}+\eta \widehat{\psi}_{-}^{\mu}\right)\right] .
\end{aligned}
$$

The vanishing of the first term implies, using the Dirichlet condition (2.19),

$$
\pi_{\nu}^{\mu} \omega_{\mu}=0
$$

which also annihilates the last term because, due to the fermionic Dirichlet condition (2.15) and $Q^{\mu}{ }_{\nu} k^{\nu}=0$, Eq. (3.2), $\widehat{\psi}_{ \pm}^{\mu}$ satisfy

$$
Q^{\mu}{ }_{\nu}\left(\widehat{\psi}_{+}^{\nu}+\eta \widehat{\psi}_{-}^{\nu}\right)=0
$$

Because (4.14) implies $k^{\mu} \omega_{\mu}=0$ and therefore $\left(k^{\mu} \omega_{\mu}\right)_{, \nu}=0$, the remaining term in (5.15) may be rewritten as

$$
i \epsilon v_{+} k^{\lambda} \pi_{\lambda}^{\mu} \omega_{[\rho, \mu]} \pi_{\nu}^{\rho}\left(\widehat{\psi}_{+}^{\nu}+\eta \widehat{\psi}_{-}^{\nu}\right)
$$

which implies

$$
k^{\lambda} \pi_{\lambda}^{\mu}\left(\mathcal{L}_{k} B_{\mu \rho}\right) \pi_{\nu}^{\rho}=0,
$$

i.e., Eq. (3.23) contracted with $k^{\mu}$.

The action (5.14) is thus gauge invariant provided (4.14), (5.16) and (5.19) are satisfied on the boundary, and we can now go to adapted coordinates $\left(k^{\mu}=\delta_{0}^{\mu}\right)$, and fix the gauge such that $\Lambda=0$. The result is the original sigma model action,

$$
S=\int d^{2} \xi d^{2} \theta \quad D_{+} \Phi^{\mu} D_{-} \Phi^{\nu} E_{\mu \nu}+\frac{i}{2} \int d \tau B_{\mu \nu}\left[\psi_{+}^{\mu} \psi_{+}^{\nu}+\psi_{-}^{\mu} \psi_{-}^{\nu}\right] .
$$

In Table 9 we collect the conditions necessary for a consistent retrieval of the original supersymmetric sigma model from the parent action (5.3), assuming the conditions in Table 8 are satisfied. 


$$
\begin{aligned}
Q_{\nu}^{\mu}{ }_{\nu} \partial_{\tau} X^{\nu} & =0 \\
Q^{\mu}{ }_{\nu} k^{\nu} & =0 \\
\mathcal{L}_{k} g_{\mu \nu} & =0 \\
\mathcal{L}_{k} B_{\mu \nu}-\omega_{[\nu, \mu]} & =0 \\
\pi^{\mu}{ }_{\nu} \omega_{\mu} & =0 \\
\epsilon_{-}-\eta \epsilon_{+} & =0 \\
Q^{\mu}{ }_{\nu}\left(\widehat{\psi}_{+}^{\nu}+\eta \widehat{\psi}_{-}^{\nu}\right) & =0 \\
k^{\lambda} \pi_{\lambda}^{\mu}{ }_{\lambda}\left(\mathcal{L}_{k} B_{\mu \rho}\right) \pi_{\nu}^{\rho} & =0
\end{aligned}
$$

Table 9: Conditions necessary for a consistent retrieval of the original supersymmetric sigma model from the parent action (5.3). We have assumed that the conditions in Table 8 are satisfied.

\subsubsection{Finding the dual model}

To find the dual model we use the bulk equations of motion (5.6) to integrate out $V_{ \pm}$in (5.3), resulting in the following action,

$$
\begin{aligned}
S_{Y} & =\int d^{2} \xi d^{2} \theta\left[D_{+} \Phi^{\mu} D_{-} \Phi^{\nu} E_{\mu \nu}\right. \\
& \left.+\left(k^{\lambda} k^{\sigma} E_{\lambda \sigma}\right)^{-1}\left(D_{+} Y+\omega_{\mu} D_{+} \Phi^{\mu}+k^{\mu} E_{\nu \mu} D_{+} \Phi^{\nu}\right)\left(D_{-} Y+\omega_{\rho} D_{-} \Phi^{\rho}-k^{\gamma} E_{\gamma \rho} D_{-} \Phi^{\rho}\right)\right] \\
& +\frac{i}{2} \int d \tau B_{\mu \nu}\left[\widehat{\psi}_{+}^{\mu} \widehat{\psi}_{+}^{\nu}+\widehat{\psi}_{-}^{\mu} \widehat{\psi}_{-}^{\nu}\right] .
\end{aligned}
$$

This action should be gauge invariant, and its gauge transformation is a pure boundary term,

$$
\begin{aligned}
\delta_{k} S_{Y}= & \int d \tau\left[2 \epsilon\left(\partial_{\tau} y+\omega_{\mu} \partial_{\tau} X^{\mu}\right)+i k^{\mu} \omega_{\mu}\left(\epsilon_{+} v_{+}+\epsilon_{-} v_{-}\right)+i \epsilon k^{\nu} \omega_{[\nu, \mu]}\left(\widehat{\psi}_{+}^{\mu} v_{+}+\widehat{\psi}_{-}^{\mu} v_{-}\right)\right. \\
& \left.-i \omega_{\mu}\left(\epsilon_{+} \widehat{\psi}_{+}^{\mu}+\epsilon_{-} \widehat{\psi}_{-}^{\mu}\right)-i\left(\epsilon_{+} y_{+}+\epsilon_{-} y_{-}\right)\right]
\end{aligned}
$$

which vanishes when we use the conditions listed in Tables 8 and 9 , showing that $S_{Y}$ is gauge invariant. Note how this requires (5.12) to be an off-shell condition.

Going to adapted coordinates and fixing the gauge such that $\Phi^{0}=0$ (and hence $X^{0}=$ $\left.\psi_{ \pm}^{0}=0\right)$, we obtain the dual action,

$$
\begin{aligned}
\widetilde{S}^{\prime} & =\int d^{2} \xi d^{2} \theta\left[D_{+} Y D_{-} Y \widetilde{E}_{00}+D_{+} Y D_{-} \Phi^{n} \widetilde{E}_{0 n}+D_{+} \Phi^{m} D_{-} Y \widetilde{E}_{m 0}+D_{+} \Phi^{m} D_{-} \Phi^{n} \widetilde{E}_{m n}\right] \\
& +\frac{i}{2} \int d \tau\left\{E_{m n}\left[\widehat{\psi}_{+}^{m} \widehat{\psi}_{+}^{n}+\widehat{\psi}_{-}^{m} \widehat{\psi}_{-}^{n}\right]+E_{0 n}\left[\widehat{\psi}_{+}^{0} \widehat{\psi}_{+}^{n}+\widehat{\psi}_{-}^{0} \widehat{\psi}_{-}^{n}\right]+E_{n 0}\left[\widehat{\psi}_{+}^{n} \widehat{\psi}_{+}^{0}+\widehat{\psi}_{-}^{n} \widehat{\psi}_{-}^{0}\right]\right\},(5.22)
\end{aligned}
$$


where $\widetilde{E}_{\mu \nu}$ is the dual background given by Buscher's rules (4.19), except now it is a superfield. To rewrite the boundary term in $\widetilde{S}^{\prime}$ in terms of dual quantities, we first observe that, in the chosen gauge,

$$
\widehat{\psi}_{ \pm}^{0} \equiv \psi_{ \pm}^{0}+v_{ \pm}=v_{ \pm}, \quad \widehat{\psi}_{ \pm}^{n} \equiv \psi_{ \pm}^{n}
$$

We moreover rewrite the component form of the $V_{ \pm}$bulk equations of motion (5.6) in terms of the dual background,

$$
\left\{\begin{array}{l}
v_{+} \widetilde{E}_{00}^{-1}+\widetilde{E}_{00}^{-1} \widetilde{E}_{n 0} \psi_{+}^{n}+y_{+}=0 \\
v_{-} \widetilde{E}_{00}^{-1}-\widetilde{E}_{00}^{-1} \widetilde{E}_{0 n} \psi_{-}^{n}-y_{-}=0 .
\end{array}\right.
$$

Given $y_{-}+\eta y_{+}=0$, Eq. (5.10), and $v_{-}-\eta v_{+}=0$, Eq. (5.12), the first equation minus $\eta \equiv \pm 1$ times the second, and restricting to the boundary, yields the relation

$$
\widetilde{E}_{n 0} \psi_{+}^{n}=-\eta \widetilde{E}_{0 n} \psi_{-}^{n}
$$

Using (5.23) and (5.25) as well as (4.14) and Buscher's rules (4.19), we may now rewrite the dual action (5.22) as

$$
\widetilde{S}=\int d^{2} \xi d^{2} \theta \quad D_{+} \widetilde{\Phi}^{\mu} D_{-} \widetilde{\Phi}^{\nu} \widetilde{E}_{\mu \nu}+\frac{i}{2} \int d \tau \widetilde{B}_{\mu \nu}\left[\widetilde{\psi}_{+}^{\mu} \widetilde{\psi}_{+}^{\nu}+\widetilde{\psi}_{-}^{\mu} \widetilde{\psi}_{-}^{\nu}\right]
$$

where

$$
\widetilde{\Phi}^{0} \equiv Y, \quad \widetilde{\Phi}^{n} \equiv \Phi^{n}, \quad \widetilde{\psi}_{ \pm}^{0} \equiv y_{ \pm}, \quad \widetilde{\psi}_{ \pm}^{n} \equiv \psi_{ \pm}^{n}
$$

Thus the dual action has the same form as the original action, with $Y$ playing the role of a $\Phi^{0}$-coordinate in the dual coordinate system, and $y_{ \pm}$being the dual analogues of $\psi_{ \pm}^{0}$.

\subsection{Gauge invariance of parent action}

It is easily verified that the gauge transformation of the parent action before integrating out the fields $V_{ \pm}$is the same as after integrating them out, i.e., Eq. (5.21). As already discussed, this vanishes when all the boundary conditions listed in Tables 8 and 9 are satisfied, hence the parent action (5.3) is indeed gauge invariant.

\subsection{Compatibility of field equations, part I}

Now we want to compare the boundary equations of motion obtained from the parent action with the bulk equations restricted to the boundary, to check if they are compatible. The boundary field equations for $Y$ were derived in Section 5.1 and are listed in Table 8. There are no boundary equations of motion for $V_{ \pm}$, so we move on to derive the $X^{\mu}$ and $\widehat{\psi}_{ \pm}^{\mu}$ field 
equations. The boundary term that results from varying the parent action (5.3) with respect to $X^{\mu}$ and $\widehat{\psi}_{ \pm}^{\mu}$ in component form reads

$$
\begin{aligned}
\delta \widehat{S}= & \int d \tau\left\{\delta X ^ { \rho } \left[\partial_{=} X^{\mu} E_{\rho \mu}-\partial_{+} X^{\mu} E_{\mu \rho}\right.\right. \\
& +i \widehat{\psi}_{+}^{\mu} v_{+}\left(\mathcal{L}_{k} B_{\mu \rho}-\omega_{\rho, \mu}\right)-i \widehat{\psi}_{-}^{\mu} v_{-}\left(\mathcal{L}_{k} B_{\rho \mu}+\omega_{\rho, \mu}\right) \\
& -i\left(v_{++}+v_{--}\right) \omega_{\rho}+i v_{++} k^{\nu} E_{\nu \rho}-i v_{--} k^{\nu} E_{\rho \nu} \\
& \left.+i \widehat{\psi}_{+}^{\mu} \widehat{\psi}_{+}^{\nu}\left(E_{\nu \rho, \mu}+\frac{1}{2} B_{\mu \nu, \rho}\right)+i \widehat{\psi}_{-}^{\mu} \widehat{\psi}_{-}^{\nu}\left(E_{\rho \mu, \nu}+\frac{1}{2} B_{\mu \nu, \rho}\right)\right] \\
& \left.+i \delta \widehat{\psi}_{+}^{\mu}\left[\widehat{\psi}_{+}^{\nu} g_{\mu \nu}-v_{+} \omega_{\mu}\right]-i \delta \widehat{\psi}_{-}^{\mu}\left[\widehat{\psi}_{-}^{\nu} g_{\mu \nu}+v_{-} \omega_{\mu}\right]\right\} .
\end{aligned}
$$

Using $\delta X^{\rho}=\delta X^{\lambda} \pi_{\lambda}^{\rho}$ as well as (3.7), (3.8) and (4.14), $\delta \widehat{S}$ reduces to

$$
\begin{aligned}
\delta \widehat{S}= & \int d \tau\left\{\delta X ^ { \lambda } \pi _ { \lambda } ^ { \rho } \left[\partial_{=} X^{\mu} E_{\rho \mu}-\partial_{+} X^{\mu} E_{\mu \rho}\right.\right. \\
& -i\left(\widehat{\psi}_{+}^{\mu} v_{+}+\widehat{\psi}_{-}^{\mu} v_{-}\right) \omega_{\mu, \rho}+i v_{++} k^{\nu} E_{\nu \rho}-i v_{--} k^{\nu} E_{\rho \nu} \\
& \left.+i \widehat{\psi}_{+}^{\mu} \widehat{\psi}_{+}^{\nu}\left(E_{\nu \rho, \mu}+\frac{1}{2} B_{\mu \nu, \rho}\right)+i \widehat{\psi}_{-}^{\mu} \widehat{\psi}_{-}^{\nu}\left(E_{\rho \mu, \nu}+\frac{1}{2} B_{\mu \nu, \rho}\right)\right] \\
& \left.+i \delta \widehat{\psi}_{+}^{\mu}\left[\widehat{\psi}_{+}^{\nu} g_{\mu \nu}-v_{+} \omega_{\mu}\right]-i \delta \widehat{\psi}_{-}^{\mu}\left[\widehat{\psi}_{-}^{\nu} g_{\mu \nu}+v_{-} \omega_{\mu}\right]\right\} .
\end{aligned}
$$

We will return to this equation to derive the most general equation of motion, but first we turn to the $V_{ \pm}$bulk equations of motion, Eq. (5.6), restricting their component form to the boundary. Taking the first equation minus $\eta$ times the second one, we obtain a superfield relation whose fermionic lowest component reads

$$
0=y_{+}+\eta y_{-}-\left(v_{+}+\eta v_{-}\right) k^{\mu} \omega_{\mu}+\widehat{\psi}_{+}^{\mu}\left(\omega_{\mu}+k^{\nu} E_{\mu \nu}\right)+\eta \widehat{\psi}_{-}^{\mu}\left(\omega_{\mu}-k^{\nu} E_{\nu \mu}\right)
$$

and its bosonic superpartner reads

$$
\begin{aligned}
0= & -2 \partial_{\tau} y-2 \omega_{\mu} \partial_{\tau} X^{\mu}-\partial_{+} X^{\mu} k^{\nu} E_{\mu \nu}+\partial_{=} X^{\mu} k^{\nu} E_{\nu \mu}+i\left(v_{++}-v_{--}\right) k^{\mu} k^{\nu} g_{\mu \nu} \\
& +i \widehat{\psi}_{+}^{\rho} \widehat{\psi}_{+}^{\nu}\left(\omega_{\nu}+k^{\mu} E_{\nu \mu}\right)_{, \rho}+i \widehat{\psi}_{-}^{\rho} \widehat{\psi}_{-}^{\nu}\left(\omega_{\nu}-k^{\mu} E_{\mu \nu}\right)_{, \rho} .
\end{aligned}
$$

Inserting (4.9), (4.14), (5.10) and the Dirichlet condition (5.18), Eqs. (5.29) and (5.30) simplify to

$$
\begin{gathered}
0=k^{\nu} E_{\mu \nu} \widehat{\psi}_{+}^{\mu}-\eta k^{\nu} E_{\nu \mu} \widehat{\psi}_{-}^{\mu} \\
0=k^{\nu}\left(\partial_{=} X^{\mu} E_{\nu \mu}-\partial_{+} X^{\mu} E_{\mu \nu}\right)+i\left(v_{++}-v_{--}\right) k^{\mu} k^{\nu} g_{\mu \nu} \\
+i \widehat{\psi}_{+}^{\rho} \widehat{\psi}_{+}^{\nu}\left(\omega_{\nu}+k^{\mu} E_{\nu \mu}\right)_{, \rho}+i \widehat{\psi}_{-}^{\rho} \widehat{\psi}_{-}^{\nu}\left(\omega_{\nu}-k^{\mu} E_{\mu \nu}\right)_{, \rho} .
\end{gathered}
$$

The $\widehat{\psi} \widehat{\psi}$-terms can be rewritten using $\mathcal{L}_{k} B_{\mu \nu}=\omega_{[\nu, \mu]}$ so that (5.32) becomes

$$
\begin{aligned}
0= & k^{\nu}\left(\partial_{=} X^{\mu} E_{\nu \mu}-\partial_{+} X^{\mu} E_{\mu \nu}\right)+i\left(v_{++}-v_{--}\right) k^{\mu} k^{\nu} g_{\mu \nu} \\
& +i \widehat{\psi}_{+}^{\mu} \widehat{\psi}_{+}^{\nu}\left(E_{\nu \rho, \mu}+\frac{1}{2} B_{\mu \nu, \rho}\right) k^{\rho}+i \widehat{\psi}_{-}^{\mu} \widehat{\psi}_{-}^{\nu}\left(E_{\rho \mu, \nu}+\frac{1}{2} B_{\mu \nu, \rho}\right) k^{\rho} \\
& +i\left(\widehat{\psi}_{+}^{\mu} \widehat{\psi}_{+}^{\nu}-\widehat{\psi}_{-}^{\mu} \widehat{\psi}_{-}^{\nu}\right) k_{, \mu}^{\rho} g_{\rho \nu} .
\end{aligned}
$$


This is as far as we get without employing a boundary ansatz for the fermions. Otherwise we cannot derive the most general equations of motion from (5.28), to see if they are compatible with (5.33). We therefore turn to the fermionic ansatz next, deducing the appropriate one in a fashion similar to the bosonic analysis in Section 4.5.

\subsection{Intermission: boundary ansatz}

For the original nonlinear sigma model (2.1) we found that the appropriate fermionic ansatz was (2.9), accompanied by certain restrictions on the gluing matrix $R^{\mu}{ }_{\nu}$ dictated by superconformal symmetry. Here it is natural to make the analogous ansatz for the gauged model,

$$
\widehat{\psi}_{-}^{\mu}=\eta R^{\mu}{ }_{\nu} \widehat{\psi}_{+}^{\nu}
$$

The form of this ansatz is also suggested by the supersymmetric generalisation of the bosonic ansatz (4.23).

In analogy with the bosonic analysis in Section 4.5, we may deduce that the gluing matrix here is the same as in the ungauged model. The currents associated to superconformal invariance are identical in form to those of the original model, as shown in Appendix D. Thus the superconformal conditions are also of the same form, and in the fixed gauge they should reduce to the conditions of the original model. But again there are no consistent conformally allowed modifications to $R^{\mu}{ }_{\nu}$.

It is important for the continued analysis to realise that because the analysis of superconformal currents is identical to that of the original model, we know that all the boundary conditions in Table 1 must hold also in the gauged model. In particular, $\pi$-integrability together with $\pi_{\nu}^{\mu} \omega_{\mu}=0$ implies $\pi_{\sigma}^{\mu}\left(\mathcal{L}_{k} B_{\mu \nu}\right) \pi_{\rho}^{\nu}=0$, Eq. (3.23), hence (5.19) is automatically satisfied. Therefore we may henceforth replace (5.19) with the condition (3.23).

Just like in Section 2.2 we can write (5.34) and its bosonic superpartner in terms of 1D superfields. The supersymmetry transformation of the fermionic ansatz reads

$$
\begin{aligned}
i \partial_{=} X^{\mu}= & i R_{\nu}^{\mu} \partial_{+} X^{\nu}-2 \eta P_{\nu}^{\mu} F_{+-}^{\nu}+2 R_{\nu, \rho}^{\mu} P_{\lambda}^{\rho} \widehat{\psi}_{+}^{\lambda} \widehat{\psi}_{+}^{\nu} \\
& -R_{\nu, \rho}^{\mu} k^{\rho} v_{+} \widehat{\psi}_{+}^{\nu}+2 v_{+}\left(-R_{\nu}^{\mu} k_{, \rho}^{\nu}+k_{, \rho}^{\mu}\right) P_{\lambda}^{\rho} \widehat{\psi}_{+}^{\lambda} \\
& +\left(R^{\mu}{ }_{\nu} k^{\nu}-k^{\mu}\right)\left(v_{++}+\eta v_{-+}\right),
\end{aligned}
$$

where we have used (5.34) as well as the boundary relation for $v_{ \pm}$, Eq. (5.12), and its superpartner condition,

$$
v_{--}+\eta v_{+-}=v_{++}+\eta v_{-+}
$$


In terms of 1D superfields, (5.34) and (5.35) are summarised in the relation

$$
\left(\nabla K^{\mu}+\widehat{S}^{\mu}\right)=R_{\nu}^{\mu}(K)\left(\nabla K^{\nu}-\widehat{S}^{\nu}\right)
$$

where

$$
\nabla K^{\mu} \equiv D K^{\mu}+k^{\mu} V_{1}, \quad \widehat{S}^{\mu} \equiv S^{\mu}+k^{\mu} V_{2},
$$

with

$$
V_{1} \equiv \frac{1}{2}\left(V_{-}+\eta V_{+}\right), \quad V_{2} \equiv \frac{1}{2}\left(V_{-}-\eta V_{+}\right) .
$$

It immediately follows that the fermionic Dirichlet condition (5.18) and its superpartner (2.19) may be written analogously to (2.22),

$$
Q_{\nu}^{\mu} \nabla K^{\nu}=0
$$

It is now easy to check that gauge invariance of the ansatz (5.37) implies that the Lie derivative of $R^{\mu}{ }_{\nu}$ must vanish,

$$
\mathcal{L}_{k} R_{\nu}^{\mu}=0
$$

This is very useful in the continued analysis, and it also leads to

$$
\mathcal{L}_{k} \pi^{\mu}{ }_{\nu}=\mathcal{L}_{k} Q^{\mu}{ }_{\nu}=0
$$

\subsection{Compatibility of field equations, part II}

Let us now use the ansatz (5.34) in the $V_{ \pm}$field equations (5.31) and (5.33). First we note that (5.31) is automatically satisfied by virtue of the superconformality conditions (2.17) and $(2.20)$. Next we use $(2.11),(3.18)$ and (5.34) to rewrite the last term in (5.33). The $V_{ \pm}$ field equation then becomes

$$
\begin{aligned}
0= & k^{\nu}\left(\partial_{=} X^{\mu} E_{\nu \mu}-\partial_{+} X^{\mu} E_{\mu \nu}\right)+i\left(v_{++}-v_{--}\right) k^{\mu} k^{\nu} g_{\mu \nu} \\
& +i \widehat{\psi}_{+}^{\mu} \widehat{\psi}_{+}^{\nu}\left(E_{\nu \rho, \mu}+\frac{1}{2} B_{\mu \nu, \rho}\right) k^{\rho}+i \widehat{\psi}_{-}^{\mu} \widehat{\psi}_{-}^{\nu}\left(E_{\rho \mu, \nu}+\frac{1}{2} B_{\mu \nu, \rho}\right) k^{\rho} \\
& -i \widehat{\psi}_{+}^{\mu} \widehat{\psi}_{+}^{\nu} R^{\gamma}{ }_{\nu} g_{\gamma \rho} R_{\mu, \lambda}^{\rho} k^{\lambda}
\end{aligned}
$$

We will do the same for the $X^{\mu}$ and $\widehat{\psi}_{ \pm}^{\mu}$ field equation (5.28), but first we need the field variation of the fermionic ansatz,

$$
\delta \widehat{\psi}_{-}^{\mu}=\eta R_{\nu}^{\mu} \delta \widehat{\psi}_{+}^{\nu}+\eta R_{\nu, \rho}^{\mu} \widehat{\psi}_{+}^{\nu} \delta X^{\rho}
$$


We use this to substitute for $\delta \widehat{\psi}_{-}^{\mu}$ in (5.28), and we moreover use (2.11) as well as (5.12) and (5.34) in the $\delta \widehat{\psi}_{+}^{\mu}$-terms. Eq. (5.28) then becomes

$$
\begin{aligned}
\delta \widehat{S}= & \int d \tau\left\{\delta X ^ { \lambda } \pi _ { \lambda } ^ { \rho } \left[\partial_{=} X^{\mu} E_{\rho \mu}-\partial_{+} X^{\mu} E_{\mu \rho}+i v_{++} k^{\nu} E_{\nu \rho}-i v_{--} k^{\nu} E_{\rho \nu}\right.\right. \\
& +i \widehat{\psi}_{+}^{\mu} \widehat{\psi}_{+}^{\nu}\left(E_{\nu \rho, \mu}+\frac{1}{2} B_{\mu \nu, \rho}\right)+i \widehat{\psi}_{-}^{\mu} \widehat{\psi}_{-}^{\nu}\left(E_{\rho \mu, \nu}+\frac{1}{2} B_{\mu \nu, \rho}\right) \\
& \left.\left.-i \widehat{\psi}_{+}^{\mu} \widehat{\psi}_{+}^{\nu} R_{\nu}^{\gamma} g_{\gamma \lambda} R_{\mu, \rho}^{\lambda}-2 i \widehat{\psi}_{+}^{\mu} v_{+}\left(P_{\mu}^{\nu} \omega_{\nu}\right)_{, \rho}\right]-2 i \delta \widehat{\psi}_{+}^{\mu} v_{+} P_{\mu}^{\nu} \omega_{\nu}\right\}
\end{aligned}
$$

The last term in the square bracket as well as the $\delta \widehat{\psi}_{+}^{\mu}$-term vanish by virtue of (4.14) since $P_{\mu}^{\nu} \omega_{\nu}=P_{\mu}^{\nu} \pi_{\nu}^{\rho} \omega_{\rho}=0$. Thus the final, most general, $X^{\mu}$ boundary equation of motion reads

$$
\begin{aligned}
0= & \pi_{\lambda}^{\rho}\left[\partial_{=} X^{\mu} E_{\rho \mu}-\partial_{+} X^{\mu} E_{\mu \rho}+i v_{++} k^{\nu} E_{\nu \rho}-i v_{--} k^{\nu} E_{\rho \nu}\right. \\
& +i \widehat{\psi}_{+}^{\mu} \widehat{\psi}_{+}^{\nu}\left(E_{\nu \rho, \mu}+\frac{1}{2} B_{\mu \nu, \rho}\right)+i \widehat{\psi}_{-}^{\mu} \widehat{\psi}_{-}^{\nu}\left(E_{\rho \mu, \nu}+\frac{1}{2} B_{\mu \nu, \rho}\right) \\
& \left.-i \widehat{\psi}_{+}^{\mu} \widehat{\psi}_{+}^{\nu} R_{\nu}^{\gamma} g_{\gamma \sigma} R_{\mu, \rho}^{\sigma}\right]
\end{aligned}
$$

It is immediately clear that (5.42) contracted with $k^{\lambda}$ is precisely Eq. (5.39), hence the $X^{\mu}$ field equations are compatible with the $V_{ \pm}$equations on the boundary, which is what we wanted to show.

\subsection{Gauge invariance of boundary conditions}

Next we check gauge invariance of all the boundary conditions used in this section. We start with the superconformal conditions in Table 1, and then turn to the conditions found in Sections 5.1-5.5. The only condition in Table 1 that is not trivially gauge invariant is the Dirichlet condition (2.22). Under a gauge transformation it becomes, using $Q^{\mu}{ }_{\nu} k^{\nu}=$ $Q^{\mu}{ }_{\nu} D K^{\nu}=0$,

$$
k^{\sigma} \pi_{\sigma}^{\rho} Q_{[\nu, \rho]}^{\mu} \pi_{\lambda}^{\nu} D K^{\lambda}=0
$$

which is satisfied due to $\pi$-integrability, Eq. (2.18). The analysis of the corresponding condition (5.38) for covariant fields is identical, and we already checked in Section 5.4 that the 
ansatz (5.37) is gauge invariant. It remains to check the following conditions:

$$
\begin{aligned}
Q^{\mu}{ }_{\nu} k^{\nu} & =0 \\
\mathcal{L}_{k} g_{\mu \nu} & =0 \\
\mathcal{L}_{k} B_{\mu \nu}-\partial_{[\mu} \omega_{\nu]} & =0 \\
\mathcal{L}_{k} R^{\mu}{ }_{\nu} & =0 \\
\mathcal{L}_{k} \pi^{\mu}{ }_{\nu} \mathcal{L}_{k} Q^{\mu}{ }_{\nu} & =0 \\
\pi^{\mu}{ }_{\lambda}\left(\mathcal{L}_{k} B_{\mu \rho}\right) \pi^{\rho}{ }_{\nu} & =0 \\
\partial_{\tau} y & =0 \\
\pi_{\nu}^{\mu} \omega_{\mu} & =0 \\
D_{+} V_{-}+D_{-} V_{+} & =0 \\
V_{+} k^{\mu} k^{\nu} E_{\mu \nu}+D_{+} \Phi^{\nu}\left(k^{\mu} E_{\nu \mu}+\omega_{\nu}\right)+D_{+} Y & =0 \\
V_{-} k^{\mu} k^{\nu} E_{\mu \nu}+D_{-} \Phi^{\nu}\left(k^{\mu} E_{\mu \nu}-\omega_{\nu}\right)-D_{-} Y & =0 \\
y_{-}+\eta y_{+} & =0 \\
v_{-}-\eta v_{+} & =0 \\
v_{--}+\eta v_{+-}-v_{++}-\eta v_{-+} & =0
\end{aligned}
$$

The requirements for gauge invariance of each of these conditions are as follows:

1. Eqs. (3.2), (3.7), (3.8), (3.18), (3.19), (3.23), (4.14) and (5.4) are trivially gauge invariant.

2. (4.9) is gauge invariant if $k^{\mu} \omega_{\mu}=0$, which follows from (4.14).

3. Eqs. (5.6) are gauge invariant due to (3.8).

4. Given (4.14), (5.10) is gauge invariant if $k^{\mu} \pi_{\mu}^{\rho} \pi_{\gamma}^{\nu} \pi_{[\rho, \nu]}^{\lambda}=0$, which follows from $\pi$ integrability, Eq. (2.18).

5. (5.12) is gauge invariant due to $\epsilon_{-}-\eta \epsilon_{+}=0$, Eq. (5.16).

6. (5.36) is gauge invariant due to the superpartner of $\epsilon_{-}-\eta \epsilon_{+}=0$,

$$
\epsilon_{--}+\eta \epsilon_{+-}-\epsilon_{++}-\eta \epsilon_{-+}=0
$$

\subsection{Summary and interpretation}

Thus all boundary conditions are gauge invariant, and we summarise the off-shell ones in Table 10. The full set of boundary conditions required for a consistent T-duality transformation of the $\mathcal{N}=1$ nonlinear sigma model are thus contained in Tables 1 and 10. The interpretation of the superconformality conditions in Table 1 is the same as in [16], i.e., the D-branes in the 


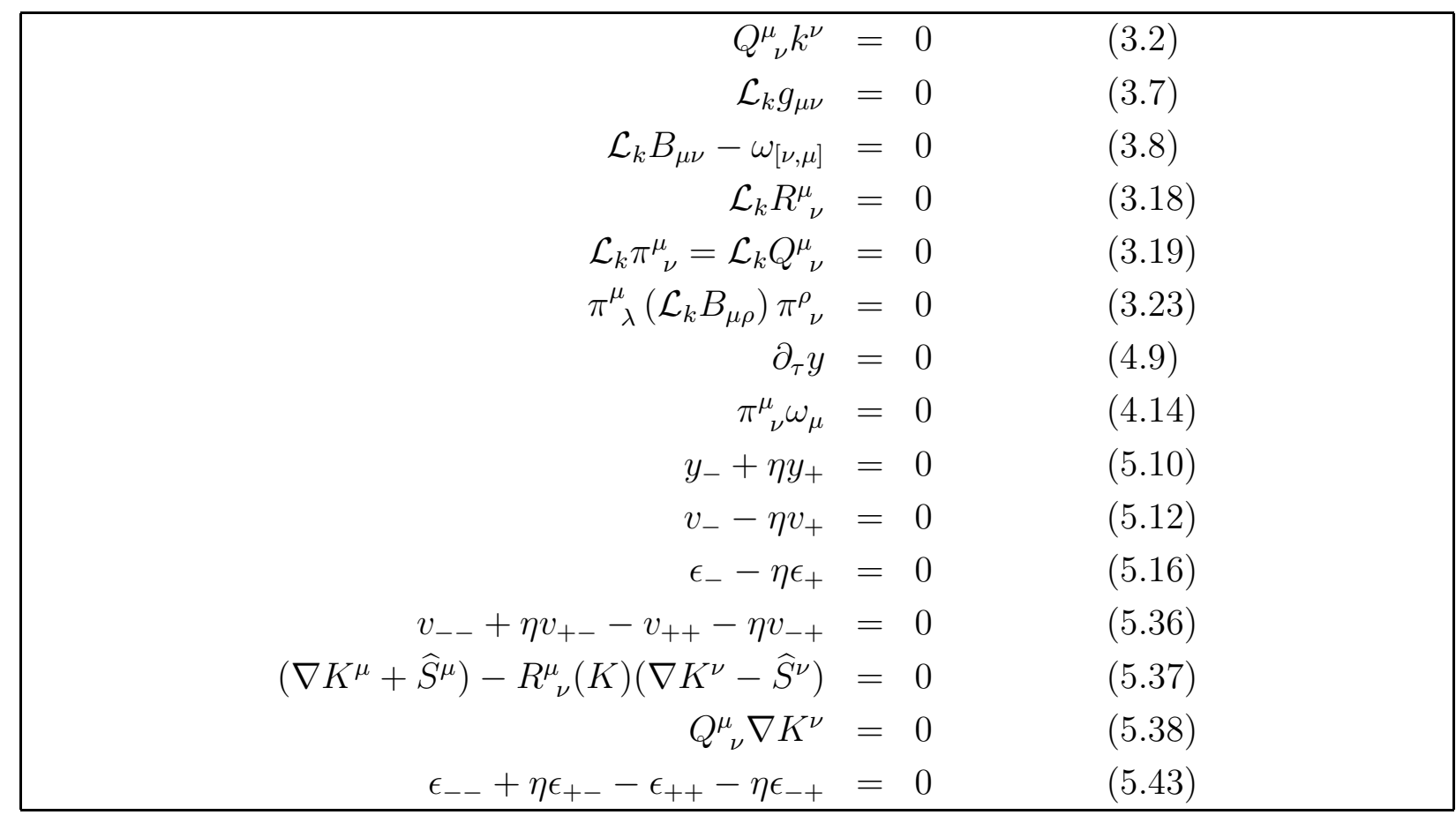

Table 10: Boundary conditions for the $\mathcal{N}=1$ supersymmetric nonlinear sigma model to be consistent under T-duality. We assume that the superconformality conditions listed in Table 1 are satisfied.

original sigma model are maximal integral submanifolds of the target space. The conditions in Table 10 are mostly the same as those required for the bosonic model (see Section 4.6) supplemented with boundary conditions for the fermionic superpartner fields. We therefore again find that the D-brane in the original model is an isometry invariant submanifold with invariant metric and two-form, and that it loses one dimension as we go to the dual model.

Eq. (5.37) is just the ansatz we made for the relation between left- and right-movers on the worldsheet, and (5.38) is the usual Dirichlet condition.

\subsection{Boundary supersymmetry}

Here we check that the parent action (5.3) is supersymmetric on the boundary. To do this, we use the conditions in Tables 1 and 10 to verify that its supersymmetry variation vanishes. The bulk part of (5.3) can be written in terms of a superfield Lagrangian $\mathcal{L}$,

$$
\widehat{S}_{\text {bulk }}=\int d^{2} \xi D_{+} D_{-} \mathcal{L} .
$$


Applying the supersymmetry transformation (A.2) we get a pure boundary term,

$$
\delta_{s} \widehat{S}_{\text {bulk }}=-i \int d \tau \epsilon^{+}\left[D_{-} \mathcal{L}+\eta D_{+} \mathcal{L}\right]
$$

The expansion of $\delta_{s} \widehat{S}_{\text {bulk }}$ in components reads

$$
\delta_{s} \widehat{S}_{\text {bulk }}=i \int d \tau \epsilon^{+}\left[\mathcal{L}_{1}+\mathcal{L}_{2}+\mathcal{L}_{3}\right]
$$

where

$$
\begin{aligned}
\mathcal{L}_{1} \equiv & \widehat{\psi}_{+}^{\mu}\left(i \partial_{=} X^{\nu}+k_{, \rho}^{\nu} \widehat{\psi}_{-}^{\rho} v_{-}+k^{\nu} v_{--}\right) E_{\mu \nu} \\
& -\eta\left(i \partial_{+} X^{\mu}+k_{, \rho}^{\mu} \widehat{\psi}_{+}^{\rho} v_{+}+k^{\mu} v_{++}\right) \widehat{\psi}_{-}^{\nu} E_{\mu \nu} \\
& +\eta \widehat{\psi}_{+}^{\mu}\left(F_{+-}^{\nu}+k_{, \rho}^{\nu} \widehat{\psi}_{+}^{\rho} v_{-}+k^{\nu} v_{+-}\right) E_{\mu \nu} \\
& -\left(-F_{+-}^{\mu}+k_{, \rho}^{\mu} \widehat{\psi}_{-}^{\rho} v_{+}+k^{\mu} v_{-+}\right) \widehat{\psi}_{-}^{\nu} E_{\mu \nu} \\
& -\widehat{\psi}_{+}^{\mu} \widehat{\psi}_{-}^{\nu}\left(\widehat{\psi}_{-}^{\rho}-k^{\rho} v_{-}\right) E_{\mu \nu, \rho}-\eta \widehat{\psi}_{+}^{\mu} \widehat{\psi}_{-}^{\nu}\left(\widehat{\psi}_{+}^{\rho}-k^{\rho} v_{+}\right) E_{\mu \nu, \rho}, \\
\mathcal{L}_{2} \equiv & -\left(\eta \widehat{\psi}_{+}^{\rho}+\widehat{\psi}_{-}^{\rho}\right) \omega_{\nu, \rho}\left(\widehat{\psi}_{+}^{\nu} v_{-}+\widehat{\psi}_{-}^{\nu} v_{+}\right) \\
& +\omega_{\nu} F_{+-}^{\nu}\left(v_{-}-\eta v_{+}\right)-i \omega_{\nu} \partial_{=} X^{\nu} v_{+}-i \eta \omega_{\nu} \partial_{+} X^{\nu} v_{-} \\
& +\omega_{\nu}\left(\widehat{\psi}_{+}^{\nu}-k^{\nu} v_{+}\right)\left(v_{--}+\eta v_{+-}\right)+\omega_{\nu}\left(\widehat{\psi}_{-}^{\nu}-k^{\nu} v_{-}\right)\left(\eta v_{++}+v_{-+}\right), \\
\mathcal{L}_{3} \equiv & v_{--} y_{+}+v_{-} y_{+-}+v_{-+} y_{-}-i v_{+} \partial_{=} y \\
& +\eta v_{++} y_{-}-\eta v_{+} y_{+-}+\eta v_{+-} y_{+}-i \eta v_{-} \partial_{+} y .
\end{aligned}
$$

For the boundary $\widehat{\psi} \hat{\psi}$-term in (5.3) we use the component form of the supersymmetry variation, Eq. (A.4). This yields the following transformation,

$$
\begin{aligned}
\delta_{s} \widehat{S}_{\widehat{\psi} \widehat{\psi}}= & i \int d \tau \epsilon^{+}\left[-i \partial_{+} X^{\mu} \widehat{\psi}_{+}^{\nu} B_{\mu \nu}-i \eta \partial_{=} X^{\mu} \widehat{\psi}_{-}^{\nu} B_{\mu \nu}+\eta F_{+-}^{\mu} B_{\mu \nu}\left(\widehat{\psi}_{+}^{\nu}-\eta \widehat{\psi}_{-}^{\nu}\right)\right. \\
& +\left(\widehat{\psi}_{+}^{\mu}+\eta \widehat{\psi}_{-}^{\mu}\right) B_{\mu \nu} k^{\nu}\left(v_{++}+\eta v_{-+}\right) \\
& -\frac{1}{2}\left(\widehat{\psi}_{+}^{\mu} \widehat{\psi}_{+}^{\nu}+\widehat{\psi}_{-}^{\mu} \widehat{\psi}_{-}^{\nu}\right) B_{\mu \nu, \rho}\left(\widehat{\psi}_{+}^{\rho}+\eta \widehat{\psi}_{-}^{\rho}\right) \\
& +v_{+}\left(\widehat{\psi}_{+}^{\mu} \widehat{\psi}_{+}^{\nu}+\widehat{\psi}_{-}^{\mu} \widehat{\psi}_{-}^{\nu}\right)\left(\mathcal{L}_{k} B_{\mu \nu}-B_{\rho \nu} k_{, \mu}^{\rho}\right) \\
& \left.+\eta v_{+} \widehat{\psi}_{+}^{\mu} \widehat{\psi}_{-}^{\nu}\left(\mathcal{L}_{k} B_{\mu \nu}-B_{\mu \nu, \rho} k^{\rho}\right)\right] .
\end{aligned}
$$

The parent action is supersymmetric if $\delta_{s} \widehat{S}_{\text {bulk }}+\delta_{s} \widehat{S}_{\widehat{\psi} \widehat{\psi}}=0$. We start by noting that $\mathcal{L}_{3}$ vanishes when we use (4.9), (5.10) and (5.12). Similarly, $\mathcal{L}_{2}$ vanishes when we use (3.23), (4.14) and (5.12). We are thus left with $\mathcal{L}_{1}$ and $\delta_{s} \widehat{S}_{\widehat{\psi} \widehat{\psi}}$. We first collect the $\partial_{=} X^{\mu}$-terms and substitute the fermionic ansatz (5.34) and its bosonic superpartner (5.35). The $\partial_{=} X^{\mu}$-terms in (5.45) plus (5.48) read

$$
i \partial_{=} X^{\mu}\left(E_{\nu \mu} \widehat{\psi}_{+}^{\nu}-\eta B_{\mu \nu} \widehat{\psi}_{-}^{\nu}\right)
$$


and after using the ansatz the total supersymmetry variation contains five different types of terms: $\partial_{+} X^{\mu}$-terms, $F_{+-}^{\mu}$-terms, $\left(v_{++}+\eta v_{-+}\right)$-terms, $v_{+} \widehat{\psi}_{+}^{\mu} \widehat{\psi}_{+}^{\nu}$-terms, and $\widehat{\psi}_{+}^{\mu} \widehat{\psi}_{+}^{\nu} \widehat{\psi}_{+}^{\rho}$-terms. It is straightforward to verify that each type of term vanishes separately when we use conditions (2.11), (2.17), (2.18), (2.20) and (3.18). A crucial relation in this computation follows from the four conditions $(2.11),(2.17),(2.18)$ and (2.20), and reads

$$
R_{\rho}^{\mu} B_{\mu \lambda} R_{\nu}^{\lambda}=-B_{\rho \nu}-E_{\rho \lambda} R_{\nu}^{\lambda}+E_{\nu \lambda} R_{\rho}^{\lambda} .
$$

We have thus shown that the total variation $\delta_{s} \widehat{S}_{\text {bulk }}+\delta_{s} \widehat{S}_{\widehat{\psi} \widehat{\psi}}$ vanishes when we use the boundary conditions listed in Tables 1 and 10. Hence the parent action is indeed supersymmetric given all the conditions derived for consistency and gauge invariance.

We also need to check that the superpartner of each of the boundary conditions holds, since the supersymmetry algebra must close. The ansatz (5.37) and the Dirichlet condition (5.38) are both manifestly supersymmetric, and for each of the other boundary conditions whose superpartner is nontrivial, its superpartner is included in the set of required conditions. This is obvious when we list them in superpartner pairs:

$$
\begin{aligned}
\partial_{\tau} y & =0 \\
y_{-}+\eta y_{+} & =0 \\
v_{-}-\eta v_{+} & =0 \\
v_{--}+\eta v_{+-}-v_{++}-\eta v_{-+} & =0 \\
\epsilon_{-}-\eta \epsilon_{+} & =0 \\
\epsilon_{--}+\eta \epsilon_{+-}-\epsilon_{++}-\eta \epsilon_{-+} & =0
\end{aligned}
$$

The rest of the conditions in Tables 1 and 10 are trivially supersymmetric, hence we have shown that the gauged model is completely supersymmetric on the boundary.

\section{Boundary couplings}

Interactions are in general described by a path integral of the form

$$
\int D \Phi(\mathcal{V} \mathcal{V} \ldots \mathcal{V}) e^{-S}
$$

where the $\mathcal{V}$ 's denote vertex operators and $S$ is the worldsheet action. The open string may couple to fields on the brane, giving rise to interactions that modify the action $S$ by boundary terms. One possible such perturbation is the standard $U(1)$ boundary coupling (using 1D superfields) $[9,30,31,32]$

$$
S_{U(1)}=\int d \tau d \theta A_{\mu}(K) D K^{\mu}=\int d \tau\left(i A_{\mu}(X) \partial_{\tau} X^{\mu}-\frac{1}{2} F_{\mu \nu}(X) \Psi^{\mu} \Psi^{\nu}\right)
$$


where $F_{\mu \nu} \equiv \partial_{[\mu} A_{\nu]}$. The fact that the $A$-field lives on the brane is expressed by the condition $A_{\mu} Q^{\mu}{ }_{\nu}=0$. Another boundary perturbation corresponds to transverse fluctuations of the brane [33],

$$
S_{\text {fluct }}=\int d \tau d \theta Y_{\mu}(K) S^{\mu}=\int d \tau\left(Y_{\mu}(X)\left(\eta F_{+-}^{\mu}-i \partial_{\sigma} X^{\mu}\right)+Y_{\mu, \nu} \Psi^{\nu} \widehat{\Psi}^{\mu}\right),
$$

where $Y_{\mu}$ is an auxiliary scalar field. Moreover, introducing the auxiliary spinor superfield $\Gamma=d+\theta Z$, one can write down a tachyonic vertex with tachyon potential $\mathcal{T}[34]$,

$$
S_{\text {tach }}=\int d \tau d \theta(\mathcal{T}(K) \Gamma+\Gamma D \Gamma)=\int d \tau\left(\frac{\partial \mathcal{T}}{\partial X^{\mu}} \Psi^{\mu} d+\mathcal{T}(X) Z+Z^{2}-i d \partial_{\tau} d\right)
$$

There are also boundary perturbations corresponding to massive open string states [35],

$$
S_{m a s s}^{(1)}=\int d \tau d \theta A_{\mu \nu}(K) D^{2} K^{\mu} D K^{\nu}
$$

where $A_{\mu \nu}$ is a massive symmetric tensor field, or

$$
S_{\text {mass }}^{(2)}=\int d \tau d \theta A_{\mu \nu \rho}(K) D K^{\mu} D K^{\nu} D K^{\rho}
$$

for a massive general tensor $A_{\mu \nu \rho}$.

Note that the boundary interactions $S_{U(1)}, S_{\text {fluct }}, S_{\text {tach }}, S_{\text {mass }}^{(1)}$ and $S_{\text {mass }}^{(2)}$ are individually supersymmetric, whereas the nonlinear sigma model (2.1) is supersymmetric only subject to the boundary conditions (2.9)-(2.12). Thus the boundary interactions are suitable for supersymmetric perturbation theory in the sense that the action (2.1) produces the supersymmetric propagators and the perturbations (6.1)-(6.5) give the supersymmetric vertices.

When modifying the worldsheet action with boundary interactions, we must check that the new action is still isometry invariant as well as consistent with T-duality. Since we know that these criteria are fulfilled by the action (2.1) separately, and we know how to gauge it in a consistent way, it remains to check that the boundary perturbations $S_{U(1)}, S_{f l u c t}, S_{\text {tach }}$, $S_{\text {mass }}^{(1)}$ and $S_{\text {mass }}^{(2)}$ are independently isometry invariant and that they can be gauged.

\subsection{Isometry invariance}

Under the isometry transformation (3.24) the $U(1)$ coupling (6.1) transforms as (recall that $\left.D \epsilon^{A}=0\right)$

$$
\delta_{k} S_{U(1)}=\epsilon^{A} \int d \tau d \theta\left(\mathcal{L}_{k_{A}} A_{\mu}\right) D K^{\mu} .
$$

For this to vanish, the Lie derivative must satisfy

$$
\pi^{\mu}{ }_{\nu} \mathcal{L}_{k_{A}} A_{\mu}=\partial_{\nu} C_{A}
$$


for some scalar field $C_{A}$. The transformation acts identically on $S_{\text {fluct }}$, resulting in the condition

$$
\pi^{\mu}{ }_{\nu} \mathcal{L}_{k_{A}} Y_{\mu}=\partial_{\nu} C_{A}
$$

For the tachyonic vertex we assume that $\Gamma$ is annihilated by an isometry transformation, $\delta_{k_{A}} \Gamma=0$, hence $S_{\text {tach }}$ transforms as

$$
\delta_{k} S_{\text {tach }}=\epsilon^{A} \int d \tau d \theta\left(\mathcal{L}_{k_{A}} \mathcal{T}\right) \Gamma,
$$

requiring

$$
\mathcal{L}_{k_{A}} \mathcal{T}=0
$$

in order to vanish. The massive case is analogous to that of $S_{U(1)}$ and we have

$$
\delta_{k} S_{\text {mass }}^{(1)}=i \epsilon^{A} \int d \tau d \theta\left(\mathcal{L}_{k_{A}} A_{\mu \nu}\right) \partial_{\tau} K^{\mu} D K^{\nu}
$$

implying

$$
\pi_{\rho}^{\mu}\left(\mathcal{L}_{k_{A}} A_{\mu \nu}\right) \pi_{\lambda}^{\nu}=\partial_{\rho} \partial_{\lambda} C_{A}
$$

For $S_{\text {mass }}^{(2)}$ we have

$$
\delta_{k} S_{\text {mass }}^{(2)}=\epsilon^{A} \int d \tau d \theta\left(\mathcal{L}_{k_{A}} A_{\mu \nu \rho}\right) D K^{\mu} D K^{\nu} D K^{\rho}
$$

implying

$$
\pi_{\sigma}^{\mu} \pi_{\lambda}^{\nu} \pi_{\gamma}^{\rho} \mathcal{L}_{k_{A}} A_{\mu \nu \rho}=\partial_{\sigma} \partial_{\lambda} \partial_{\gamma} C_{A} .
$$

Note that the last statement effectively declares that

$$
\pi^{\mu}{ }_{\sigma} \pi_{\lambda}^{\nu} \pi^{\rho}{ }_{\gamma} \mathcal{L}_{k_{A}} A_{\mu \nu \rho}=0
$$

since only the antisymmetric piece of $A_{\mu \nu \rho}$ is relevant in $S_{m a s s}^{(2)}$.

In conclusion, the boundary couplings must satisfy (6.6)-(6.10) for the resulting worldsheet action to be isometry invariant.

\section{$6.2 \quad$ T-duality of vertices}

We now promote the boundary actions $S_{U(1)}, S_{\text {fluct }}, S_{\text {tach }}, S_{\text {mass }}^{(1)}$ and $S_{\text {mass }}^{(2)}$ to parent actions by adding fields to make them invariant under local gauge transformations (after gauging a $U(1)$ isometry). Starting with the $U(1)$ coupling and taking $D \epsilon \neq 0$, we find that the action

$$
\widehat{S}_{U(1)}=\int d \tau d \theta A_{\mu}(K) \nabla K^{\mu}
$$

is gauge invariant if

$$
\nabla K^{\mu} \equiv D K^{\mu}+k^{\mu} V_{1}, \quad \delta_{k} V_{1}=-D \epsilon
$$


This is in complete analogy with the gauged form of the nonlinear sigma model. The parent action for $S_{\text {fluct }}$ is almost identical:

$$
\widehat{S}_{f l u c t}=\int d \tau d \theta Y_{\mu}(K) \widehat{S}^{\mu}
$$

where

$$
\widehat{S}^{\mu} \equiv S^{\mu}+k^{\mu} V_{2}, \quad \delta_{k} V_{2}=-D \epsilon
$$

The tachyonic action, however, does not receive any corrections since a gauge transformation of $S_{\text {tach }}$ leaves no $D \epsilon$-terms. Finally, the massive vertices are analogous to the $U(1)$ coupling, producing the following parent actions:

$$
\widehat{S}_{m a s s}^{(1)}=\int d \tau d \theta A_{\mu \nu} \nabla_{\tau} K^{\mu} \nabla K^{\nu}
$$

where

$$
\nabla_{\tau} K^{\mu} \equiv i \partial_{\tau} K^{\mu}+k^{\mu} D V_{1}
$$

and

$$
\widehat{S}_{m a s s}^{(2)}=\int d \tau d \theta A_{\mu \nu \rho} \nabla K^{\mu} \nabla K^{\nu} \nabla K^{\rho}
$$

One may now write the boundary interactions as

$$
\int D \Phi D V_{ \pm}(\mathcal{V} \mathcal{V} \ldots \mathcal{V}) e^{-S[\Phi, V]}
$$

where $S[\Phi, V]$ is the gauged action (5.3) plus any of the boundary interactions $\widehat{S}_{U(1)}, \widehat{S}_{f l u c t}$, $\widehat{S}_{\text {tach }}, \widehat{S}_{\text {mass }}^{(1)}$ or $\widehat{S}_{\text {mass }}^{(2)}$. The T-dual vertices ${ }^{12}$ are obtained by plugging in the bulk values for the fields $V_{1,2}$ and fixing the gauge.

\section{$7 \quad$ T-duality of boundary conditions}

Up to this point we have obtained the action T-dual to the supersymmetric nonlinear sigma model, and the boundary conditions necessary to ensure a consistent dualisation procedure. There is one more aspect to investigate: How do the boundary conditions themselves transform under T-duality? Are there any further conditions arising from such a consideration? This is a topic for extensive analysis and we will only comment on it briefly here, deferring a more detailed discussion to subsequent work.

We start by examining the transformation properties of the worldsheet fields. We look at the bosonic model here; the supersymmetric case is completely analogous and yields the

\footnotetext{
${ }^{12}$ For another discussion of T-duality of boundary couplings, see [36].
} 
same results. The isometry group is again $U(1)$ generated by a Killing vector $k^{\mu}$. The worldsheet fields transform under T-duality according to the equations of motion (4.6) after fixing the gauge. The resulting transformation can be written in a compact form in terms of matrices $\mathbf{Q}_{ \pm}$acting on the worldsheet fields (schematically) as follows [37],

$$
\left(\begin{array}{c}
\partial_{+} \widetilde{X}^{0} \\
\partial_{+} \widetilde{X}^{n}
\end{array}\right)=\mathbf{Q}_{+}\left(\begin{array}{c}
\partial_{+} X^{0} \\
\partial_{+} X^{n}
\end{array}\right), \quad\left(\begin{array}{c}
\partial_{=} \widetilde{X}^{0} \\
\partial_{=} \widetilde{X}^{n}
\end{array}\right)=\mathbf{Q}_{-}\left(\begin{array}{c}
\partial_{=} X^{0} \\
\partial_{=} X^{n}
\end{array}\right)
$$

where the matrices $\mathbf{Q}_{ \pm}$are defined as

$$
\mathbf{Q}_{+} \equiv\left(\begin{array}{cc}
-E_{00} & -E_{n 0}-\omega_{n} \\
0 & \mathbb{I}
\end{array}\right), \quad \mathbf{Q}_{-} \equiv\left(\begin{array}{cc}
E_{00} & E_{0 n}-\omega_{n} \\
0 & \mathbb{I}
\end{array}\right)
$$

and $\mathbb{I I}$ is the identity matrix.

We know that conformal invariance requires the boundary condition

$$
\partial_{=} X^{\mu}=R_{\nu}^{\mu} \partial_{+} X^{\nu}
$$

with $R^{\mu}{ }_{\nu}$ satisfying

$$
g_{\mu \nu}=R_{\mu}^{\rho} g_{\rho \sigma} R_{\nu}^{\sigma}
$$

One can perform a direct T-duality transformation of these conditions by applying (7.1), and because the dual action should also be conformally invariant the dual counterparts of (3.17) and (2.11) must be identical in form, i.e., we expect

$$
\partial_{=} \widetilde{X}^{\mu}=\widetilde{R}_{\nu}^{\mu} \partial_{+} \widetilde{X}^{\nu}, \quad \widetilde{g}_{\mu \nu}=\widetilde{R}_{\mu}^{\rho} \widetilde{g}_{\rho \sigma} \widetilde{R}_{\nu}^{\sigma} .
$$

The transformation of (3.17) reads (schematically)

$$
\partial_{=} \widetilde{X}=\mathbf{Q}_{-} R \mathbf{Q}_{+}^{-1} \partial_{+} \widetilde{X}
$$

leading us to the conclusion that

$$
\widetilde{R}=\mathbf{Q}_{-} R \mathbf{Q}_{+}^{-1}
$$

Given this relation, the transformation of (2.11),

$$
\left(\mathbf{Q}_{+}^{-1}\right)^{t} g \mathbf{Q}_{+}^{-1}=\widetilde{R}^{t}\left(\mathbf{Q}_{-}^{-1}\right)^{t} g \mathbf{Q}_{-}^{-1} \widetilde{R}
$$

implies

$$
\widetilde{g}=\left(\mathbf{Q}_{+}^{-1}\right)^{t} g \mathbf{Q}_{+}^{-1}=\left(\mathbf{Q}_{-}^{-1}\right)^{t} g \mathbf{Q}_{-}^{-1},
$$

which is just a different way of writing Buscher's rules (4.19).

For a complete treatment, the rest of the boundary conditions in Table 1 should be similarly analysed, and the T-duality transformation for the projectors $\pi^{\mu}{ }_{\nu}$ and $Q^{\mu}{ }_{\nu}$ should be derived. This will be done elsewhere. 


\section{Conclusions}

We have shown how to gauge a $U(1)$ isometry in the $\mathcal{N}=1$ supersymmetric nonlinear sigma model with boundaries in a consistent way to obtain its T-dual via a gauge invariant parent action. We have derived the most general boundary conditions that must be satisfied by the worldsheet fields in order for the duality transformation to be consistent. The bosonic model was examined first, and we found that it requires a restricted form of integrability, which can be interpreted in terms of a D-brane whose projection onto the isometry direction in the original model is an integral submanifold of the target space. The D-brane is also an isometry invariant submanifold equipped with invariant geometrical data.

For the supersymmetric sigma model we derived the conditions necessary for the isometry group to be a symmetry of the original action, and then defined the appropriate parent action, finding the boundary conditions required for it to be consistent at the level of field equations as well as gauge invariant and supersymmetric. The resulting conditions are the same as for the ungauged sigma model (see Table 1), plus a number of restrictions on the background and the added gauge fields and lagrange multipliers (see Table 10). The interpretation of the conditions in Table 1 is exactly that given in [16], i.e., the D-brane in the original model is a maximal integral submanifold of the target space. The conditions in Table 10 encode the transition of the Neumann isometry direction in the original model to a Dirichlet direction in the dual model, together with the requirement that the background should be independent of the isometry direction for the procedure to work. They imply that the D-brane is again an invariant submanifold with invariant metric and two-form.

We moreover showed how to incorporate couplings to boundary states in the T-duality transformation, and briefly discussed how the boundary conditions themselves transform under T-duality, an issue that deserves a more detailed analysis.

For a complete picture, future studies should include sigma models with more general abelian and nonabelian isometry groups, as well as models without isometries. In the latter case the gauging procedure is unsuitable for performing T-duality, and one has to resort to other, more involved methods, e.g., Poisson-Lie T-duality. In addition to classical T-duality, quantum aspects should also be investigated. This involves extending the analysis to string worldsheets of more complicated topology.

Acknowledgements: We are grateful to Martin Roček for discussions and comments. CA acknowledges support by Deutsche Forschungsgemeinschaft (DFG). UL acknowledges support by VR grant 650-1998368. MZ acknowledges support by EU grant MEIF_CT-2004500267. 


\section{A $(1,1)$ supersymmetry}

Throughout the paper we use $\mu, \nu, \ldots$ as spacetime indices, $(+,=)$ as worldsheet indices (in lightcone coordinates $\xi^{ \pm} \equiv \tau \pm \sigma$, where $\tau, \sigma$ are the usual worldsheet coordinates), and $(+,-)$ as two-dimensional spinor indices. We also use superspace conventions where the pair of spinor coordinates are labelled $\theta^{ \pm}$, and the covariant derivatives $D_{ \pm}$and supersymmetry generators $Q_{ \pm}$satisfy

$$
\begin{aligned}
& D_{+}^{2}=i \partial_{+}, \quad D_{-}^{2}=i \partial_{=}, \quad\left\{D_{+}, D_{-}\right\}=0 \\
& Q_{ \pm}=-D_{ \pm}+2 i \theta^{ \pm} \partial_{\underline{\underline{H}}}
\end{aligned}
$$

where $\partial_{\underline{\#}}=\partial_{\tau} \pm \partial_{\sigma}$. In terms of the covariant derivatives, a supersymmetry transformation of a superfield $\Phi$ is given by

$$
\begin{aligned}
\delta \Phi & \equiv\left(\epsilon^{+} Q_{+}+\epsilon^{-} Q_{-}\right) \Phi \\
& =-\left(\epsilon^{+} D_{+}+\epsilon^{-} D_{-}\right) \Phi+2 i\left(\epsilon^{+} \theta^{+} \partial_{+}+\epsilon^{-} \theta^{-} \partial_{=}\right) \Phi,
\end{aligned}
$$

where $\epsilon^{ \pm}$are the left- and right-moving supersymmetry parameters. The components of a superfield $\Phi$ are defined via projections as follows,

$$
\Phi\left|\equiv X, \quad D_{ \pm} \Phi\right| \equiv \psi_{ \pm}, \quad D_{+} D_{-} \Phi \mid \equiv F_{+-}
$$

where a vertical bar denotes "the $\theta=0$ part of". Thus, in components, the $(1,1)$ supersymmetry transformations are given by

$$
\left\{\begin{array}{l}
\delta X^{\mu}=-\epsilon^{+} \psi_{+}^{\mu}-\epsilon^{-} \psi_{-}^{\mu} \\
\delta \psi_{+}^{\mu}=-i \epsilon^{+} \partial_{+} X^{\mu}-\epsilon^{-} F_{-+}^{\mu} \\
\delta \psi_{-}^{\mu}=-i \epsilon^{-} \partial_{=} X^{\mu}-\epsilon^{+} F_{+-}^{\mu} \\
\delta F_{+-}^{\mu}=-i \epsilon^{+} \partial_{+} \psi_{-}^{\mu}+i \epsilon^{-} \partial_{=} \psi_{+}^{\mu}
\end{array}\right.
$$

\section{B 1D superfield formalism}

One may view the 2D supersymmetry algebra as a combination of two 1D algebras (for similar considerations see $[10,25])$. To see this, we rewrite the $(1,1)$ supersymmetry algebra in terms of $1 \mathrm{D}$ supermultiplets. Defining $\epsilon \equiv \epsilon^{-}$and assuming that $\epsilon^{+}=\eta \epsilon,(\mathrm{A} .4)$ becomes

$$
\left\{\begin{array}{l}
\delta X^{\mu}=-\epsilon\left(\eta \psi_{+}^{\mu}+\psi_{-}^{\mu}\right) \\
\delta\left(\eta \psi_{+}^{\mu}+\psi_{-}^{\mu}\right)=-2 i \epsilon \partial_{\tau} X^{\mu} \\
\delta\left(\psi_{-}^{\mu}-\eta \psi_{+}^{\mu}\right)=-2 \epsilon\left(\eta F_{+-}^{\mu}-i \partial_{\sigma} X^{\mu}\right) \\
\delta\left(\eta F_{+-}^{\mu}-i \partial_{\sigma} X^{\mu}\right)=-i \epsilon \partial_{\tau}\left(\psi_{-}^{\mu}-\eta \psi_{+}^{\mu}\right)
\end{array}\right.
$$


Introducing a new notation for the following combinations of fields,

$$
\Psi^{\mu} \equiv \frac{1}{\sqrt{2}}\left(\psi_{-}^{\mu}+\eta \psi_{+}^{\mu}\right), \quad \widehat{\Psi}^{\mu} \equiv \frac{1}{\sqrt{2}}\left(\psi_{-}^{\mu}-\eta \psi_{+}^{\mu}\right), \quad f^{\mu} \equiv \eta F_{+-}^{\mu}-i \partial_{\sigma} X^{\mu}
$$

and redefining $\epsilon=\sqrt{2} \epsilon$, the algebra (B.1) takes on the simple form

$$
\left\{\begin{array}{l}
\delta X^{\mu}=-\epsilon \Psi^{\mu} \\
\delta \Psi^{\mu}=-i \epsilon \partial_{\tau} X^{\mu} \\
\delta \widehat{\Psi}^{\mu}=-\epsilon f^{\mu} \\
\delta f^{\mu}=-i \epsilon \partial_{\tau} \widehat{\Psi}^{\mu}
\end{array}\right.
$$

Clearly, (B.3) is a decomposition of the 2D algebra into two 1D supermultiplets. We introduce a 1D superfield notation for these multiplets,

$$
K^{\mu}=X^{\mu}+\theta \Psi^{\mu}, \quad S^{\mu}=\widehat{\Psi}^{\mu}+\theta f^{\mu},
$$

where $\theta$ is the single Grassmann coordinate of the respective 1D superspace, and the corresponding $1 \mathrm{D}$ superderivative is now $D$, satisfying $D^{2}=i \partial_{\tau}$.

\section{The Lie derivative}

The Lie derivative with respect to a vector $k$, acting on an $(l, s)$-tensor $T$, is defined, in coordinate components, as

$$
\left(\mathcal{L}_{k} T\right)_{\nu_{1} \nu_{2} \ldots \nu_{s}}^{\mu_{1} \mu_{2} \ldots \mu_{l}}=\underset{\nu_{1} \nu_{2} \ldots \nu_{s}, \rho}{\mu_{1} \mu_{2} \ldots \mu_{l}} k^{\rho}-T_{\nu_{1} \nu_{2} \ldots \nu_{s}}^{\rho \mu_{2} \ldots \mu_{l}} k_{, \rho}^{\mu_{1}}-\ldots+T_{\rho \nu_{2} \ldots \nu_{s}}^{\mu_{1} \mu_{2} \ldots \mu_{l}} k_{, \nu_{1}}^{\rho}+\ldots
$$

\section{Supercurrents of the gauged model}

Here we write down the currents corresponding to superconformal invariance of the parent action (5.3) for the special case where $\mathcal{L}_{k} B_{\mu \nu}=0$. They are required to satisfy the following boundary conditions,

$$
T_{++}-T_{--}=0, \quad G_{+}-\eta G_{-}=0
$$

where the components are defined as follows,

$$
\begin{gathered}
G_{+}=T_{+}^{-}\left|, \quad G_{-}=T_{=}^{+}\right|, \\
T_{++}=-i D_{+} T_{+}^{-}\left|, \quad T_{--}=-i D_{-} T_{=}^{+}\right|,
\end{gathered}
$$


with supercurrent components given by

$$
\begin{aligned}
& T_{+}^{-} \equiv-\frac{i}{2} D_{+}\left(\mathcal{D}_{+} \Phi^{\mu} \mathcal{D}_{+} \Phi^{\nu} E_{\mu \nu}\right)+\mathcal{D}_{+} \Phi^{\mu} \mathcal{D}_{+} \Phi^{\nu} E_{\mu \nu}, \\
& T_{=}^{+} \equiv \quad \frac{i}{2} D_{-}\left(\mathcal{D}_{-} \Phi^{\mu} \mathcal{D}_{-} \Phi^{\nu} E_{\mu \nu}\right)+\mathcal{D}_{=} \Phi^{\mu} \mathcal{D}_{-} \Phi^{\nu} E_{\mu \nu} \text { ， }
\end{aligned}
$$

or, expanding the first term in each current,

$$
\begin{aligned}
& T_{+}^{-}=\mathcal{D}_{+} \Phi^{\mu} \mathcal{D}_{+} \Phi^{\nu} g_{\mu \nu}-\frac{i}{2} \mathcal{D}_{+} \Phi^{\mu} \mathcal{D}_{+} \Phi^{\nu} D_{+} \Phi^{\rho} E_{\mu \nu, \rho} \\
& T_{=}^{+}=\mathcal{D}_{-} \Phi^{\mu} \mathcal{D}_{=} \Phi^{\nu} g_{\mu \nu}+\frac{i}{2} \mathcal{D}_{-} \Phi^{\mu} \mathcal{D}_{-} \Phi^{\nu} D_{-} \Phi^{\rho} E_{\mu \nu, \rho}
\end{aligned}
$$

The supercurrents are conserved,

$$
D_{+} T_{=}^{+}=0, \quad D_{-} T_{+}^{-}=0 .
$$

Here we have used the following notation,

$$
\begin{aligned}
& \mathcal{D}_{ \pm} \Phi^{\mu} \equiv D_{ \pm} \Phi^{\mu}+\delta^{\mu 0} V_{ \pm}, \\
& \mathcal{D}_{\underline{\underline{\#}}} \Phi^{\mu} \equiv \partial_{\underline{\underline{\#}}} \Phi^{\mu}-i \delta^{\mu 0} V_{\underline{\underline{\#}}},
\end{aligned}
$$

where

$$
V_{+} \equiv D_{+} V_{+}, \quad V_{=} \equiv D_{-} V_{-},
$$

and the derivatives satisfy the following relations,

$$
\begin{array}{cr}
D_{ \pm}^{2}=i \partial_{\underline{\underline{H}}}, & \mathcal{D}_{ \pm}^{2}=i \mathcal{D}_{\underline{\underline{H}}} \\
\left\{D_{+}, D_{-}\right\}=0, & \left\{\mathcal{D}_{+}, \mathcal{D}_{-}\right\}=0 \\
D_{+} \mathcal{D}_{+} \Phi^{\mu}=i \mathcal{D}_{+} \Phi^{\mu}, & D_{-} \mathcal{D}_{-} \Phi^{\mu}=i \mathcal{D}_{=} \Phi^{\mu} \\
\mathcal{D}_{+} \mathcal{D}_{ \pm} \Phi^{\mu}=\mathcal{D}_{ \pm} \mathcal{D}_{+} \Phi^{\mu}=\partial_{+} \mathcal{D}_{ \pm} \Phi^{\mu}
\end{array}
$$

The currents (D.5) can be written on the more symmetric form $\left(H_{\mu \nu \rho}\right.$ is the field strength of the B-field)

$$
\begin{aligned}
& T_{+}^{-}=\mathcal{D}_{+} \Phi^{\mu} \mathcal{D}_{+} \Phi^{\nu} g_{\mu \nu}-\frac{i}{3} \mathcal{D}_{+} \Phi^{\mu} \mathcal{D}_{+} \Phi^{\nu} \mathcal{D}_{+} \Phi^{\rho} H_{\mu \nu \rho}, \\
& T_{=}^{+}=\mathcal{D}_{-} \Phi^{\mu} \mathcal{D}_{=} \Phi^{\nu} g_{\mu \nu}+\frac{i}{3} \mathcal{D}_{-} \Phi^{\mu} \mathcal{D}_{-} \Phi^{\nu} \mathcal{D}_{-} \Phi^{\rho} H_{\mu \nu \rho}
\end{aligned}
$$

so we see that they are obtained from the "ungauged" supercurrents (see [16]) simply by replacing ordinary superderivatives $D_{ \pm}$with covariant ones, $\mathcal{D}_{ \pm}$. These gauged currents reduce to those of the original and dual sigma models respectively, when we integrate out the appropriate fields ( $Y$ and $V_{ \pm}$, respectively).

\section{References}

[1] U. Lindström and M. Roček, "Scalar Tensor Duality And N=1, N=2 Nonlinear Sigma Models," Nucl. Phys. B 222 (1983) 285 
[2] C. M. Hull and B. Spence, "The gauged nonlinear sigma model with Wess-Zumino term," Phys. Lett. B 232 (1989) 204-210

[3] C. M. Hull and B. Spence, "The geometry of the gauged sigma-model with WessZumino term," Nucl. Phys. B 353 (1991) 379-426

[4] T. H. Buscher, "A symmetry of the string background field equations," Phys. Lett. B 194 (1987) 59

[5] T. H. Buscher, "Path integral derivation of quantum duality in nonlinear sigma models," Phys. Lett. B 201 (1988) 466

[6] E. Alvarez, L. Alvarez-Gaume, J. L. F. Barbon and Y. Lozano, "Some Global Aspects of Duality in String Theory," Nucl. Phys. B 415 (1994) 71-100, hep-th/9309039

[7] X. C. de la Ossa and F. Quevedo, "Duality Symmetries from Non-Abelian Isometries in String Theories," Nucl. Phys. B 403 (1993) 377-394, hep-th/9210021

[8] H. Ooguri, Y. Oz and Z. Yin, "D-branes on Calabi-Yau spaces and their mirrors," Nucl. Phys. B 477 (1996) 407, hep-th/9606112

[9] J. Borlaf and Y. Lozano, "Aspects of T-duality in open strings," Nucl. Phys. B 480 (1996) 239-264, hep-th/9607051

[10] K. Hori, "Linear Models of Supersymmetric D-Branes," hep-th/0012179

[11] C. M. Hull, A. Karlhede, U. Lindström and M. Roček, "Nonlinear $\sigma$-models and their gauging in and out of superspace," Nucl. Phys. B 266 (1986) 1-44

[12] M. Roček and E. Verlinde, "Duality, Quotients, and Currents," Nucl. Phys. B 373 (1992) 630-646, hep-th/9110053

[13] S. F. Hassan, "O $(\mathrm{d}, \mathrm{d} ; \mathrm{R})$ Deformations of Complex Structures and Extended Worldsheet Supersymmetry," Nucl. Phys. B 454 (1995) 86-102, hep-th/9408060

[14] E. Alvarez, J.L.F. Barbón and J. Borlaf, "T-duality for open strings," Nucl. Phys. B 479 (1996) 218-242, hep-th/9603089

[15] P. Haggi-Mani, U. Lindström and M. Zabzine, "Boundary conditions, supersymmetry and A-field coupling for an open string in a B-field background," Phys. Lett. B 483, (2000) 443, hep-th/0004061

[16] C. Albertsson, U. Lindström and M. Zabzine, " $\mathrm{N}=1$ supersymmetric sigma model with boundaries, II," Nucl. Phys. B 678 (2004) 295-316, hep-th/0202069 
[17] U. Lindström and M. Zabzine, "N=2 boundary conditions for non-linear sigma models and Landau-Ginzburg models," JHEP 0302 (2003) 006, hep-th/0209098

[18] U. Lindström and M. Zabzine, "D-branes in N=2 WZW models," Phys. Lett. B 560 (2003) 108-115, hep-th/0212042

[19] M. Zabzine, "Geometry of D-branes for general $\mathrm{N}=(2,2)$ sigma models," to appear in Lett. Math. Phys., hep-th/0405240

[20] D. V. Vassilevich, "Spectral branes," JHEP 0103 (2001) 023, hep-th/0102091

[21] A. Fotopoulos, "On $\left(\alpha^{\prime}\right)^{2}$ corrections to the D-brane action for non-geodesic worldvolume embeddings," JHEP 0109 (2001) 005, hep-th/0104146

[22] J. Polchinski, "TASI Lectures on D-Branes," hep-th/9611050

[23] K. Yano, "Differential geometry on complex and almost complex spaces" (Pergamon, Oxford, 1965)

[24] K. Yano and M. Kon, "Structures on manifolds," Series in Pure Mathematics, Vol.3 (World Scientific, Singapore, 1984)

[25] P. Koerber, S. Nevens and A. Sevrin, "Supersymmetric non-linear sigma-models with boundaries revisited," JHEP 0311 (2003) 066, hep-th/0309229

[26] C. Albertsson, U. Lindström and M. Zabzine, "N=1 supersymmetric sigma model with boundaries, I," Commun. Math. Phys. 233 (2003) 403-421, hep-th/0111161

[27] C. Albertsson, U. Lindström and M. Zabzine, "Superconformal boundary conditions for the WZW model," JHEP 0305 (2003) 050, hep-th/0304013

[28] U. Lindström, M. Roček and P. van Nieuwenhuizen, "Consistent boundary conditions for open strings," Nucl. Phys. B 662 (2003) 147-169, hep-th/0211266

[29] B. de Wit, C. M. Hull and M. Roček, "New topological terms in gauge invariant actions," Phys. Lett. B 184 (1987) 233-238

[30] A. A. Tseytlin, "Vector field effective action in the open superstring theory," Nucl. Phys. B 276 (1986) 391

[31] O. D. Andreev and A. A. Tseytlin, "Generating functional for scattering amplitudes and effective action in the open superstring theory," Phys. Lett. B 207 (1988) 157 
[32] O. D. Andreev and A. A. Tseytlin, "Partition function representation for the open superstring effective action: Cancellation of mobius infinities and derivative corrections to Born-Infeld lagrangian," Nucl. Phys. B 311 (1988) 205

[33] S. Hellerman, S. Kachru, A. Lawrence and J. McGreevy, "Linear Sigma Models for Open Strings," JHEP 0207 (2002) 002, hep-th/0109069

[34] J. A. Harvey, D. Kutasov and E. J. Martinec, "On the relevance of tachyons," hepth/0003101

[35] S. Frolov, "On off-shell structure of open string sigma model," JHEP 0108 (2001) 020, hep-th/0104042

[36] S. F. Hassan, " $\mathrm{N}=1$ worldsheet boundary couplings and covariance of non-Abelian worldvolume theory," hep-th/0308201

[37] S. F. Hassan, "T-Duality and Non-Local Supersymmetries," Nucl. Phys. B 460 (1996) 362-378, hep-th/9504148 